\title{
TJBF INTERNATIONAL ASSET PRICING MODELS: THE CASE OF ASEAN STOCK MARKETS
}

\author{
Chee-wooi Hooy and Kim-leng Goh \\ University Science Malaysia and \\ The University of Malaya
}

\begin{abstract}
This paper is about the role of economic grouping as it affects international capital asset pricing models, ICAPM. The conventional ICAPM is extended to include the economic grouping, regional and world factors. Inclusion of the economic grouping factor increases the explanatory power of the asset pricing models. Data on ASEAN (Indonesia, Malaysia, Philippines, Singapore and Thailand) stock markets are used in tests of the proposed models. The economic grouping factor turned out to be most important while the regional factor is least important for asset pricing in these stock markets. While four of the markets have higher systematic risk exposure to the economic group, the Singapore market, the largest market, exhibits higher exposure to world risk. The segmentation of emerging markets offers a possible explanation for these results.
\end{abstract}

Keywords: CAPM, GARCH, integration, market risk, trading bloc JEL classification: F360, G120

\section{Introduction}

The decade of the 1990 s witnessed a surge in regional trade agreements. ${ }^{1}$ Some proponents of trade regionalism argue that, since trading blocs involve lesser number of participants in the process of liberalization, blocs offer a more efficient way of moving towards globalization. Frankel et al. (1995) and Frankel and Wei (1998) suggest that the recent trade regionalisms are likely to be welfareimproving. The formation of regional trading blocs not only induces intra-trade among the member countries leading to higher economic integration, but also stimulates active capital mobilization through FDI and portfolio investment flows across capital markets. This is usually supported by the regulation and policy cooperation in the financial system and the increased likelihood of coordination in monetary and exchange rate policies. The experience of European countries

\footnotetext{
1 http://www.cid.harvard.edu/cidtrade/issues/regionalism.html.
} 
in the formation of the European Union (EU) provides a supporting case (Fratzscher, 2002).

When the formation of a trading bloc opens up trade opportunities, newly created economic activities boost prosperity of the trading region while also attracting capital inflows from member countries. What started as a trading bloc often leads to wider cooperation among eonomic grouping. Improved liquidity and market depth encourage monetary integration and a closer economic linkage among the member countries, in particular in the financial sector.

Many studies in the literature focused on the issues of portfolio diversification, market liberalization and contagion effect of an economic or financial crisis in their analysis of stock market linkages among member countries of groups. One of the early studies is by Lessard (1973) who investigated the diversification opportunity in an investment union formed by four Latino countries. That study documented evidence that the stock markets of the union members have closer relation compared to that of non-member countries. The formation of the EU has generated research interests on its capital markets some two decades later. Akdogan (1992), for example, found evidence of integration among the stock markets of EU members, suggesitng increasing relaxation of capital controls among the union members. That result is further supported by studies of Johnson and Soenen (1993) and Johnson et al. (1994) using covariance and correlation analysis.

A number of studies investigated linkages of markets in other regional blocs. Soydemir (2000) documented that the Mexican stock market is weakly linked to the markets of Argentina and Brazil, but has strong ties with the US market. The result reveals the simple fact of different trading bloc memberships - Mexico is a member of NAFTA (North American Free Trade Agreement) that includes the US, while the other two Latino markets are members of MERCOSUR (Mercado Comun del Cono Sur). The paper concludes that stock market interdependence is a result of economic convergence. Similarly, Chen et al. (2002) established evidence of a long-run equilibrium relationship among six Latino markets, attributing their findings to the formation of MERCOSUR. Although Mexico is included in their analysis, the degree of exogeneity of its market is higher, and has a weaker linkage with the other markets. More recently, Johnson and Soenen (2003) reported significant simultaneous responses that exist between Canada and Mexico (both NAFTA members) and among Argentina, Brazil, Chile (which are members of MERCOSUR) and Peru.

The literature provides clear indication that stock markets are systematically linked to their counterparts belonging to the same economic grouping. Such linkages would in turn affect the way assets are priced in these markets. On the latter aspect, however, there is little evidence in the literature documenting how economic grouping affects the asset pricing mechanism.

This study investigated the economic grouping effects on asset pricing in the case of ASEAN (Association of Southeast Asian Nations). The ASEAN Free Trade Area, or AFTA, was established with the aim of promoting intra-regional trade. Subsequent developments led to wider economic cooperation among 
ASEAN members. The concept of an ASEAN Investment Area was endorsed in order to lower barriers to intra-regional investment, to liberalize regulations, and to streamline incentives to be offered to boost regional investments. Efforts are also underway for moving towards a higher degree of monetary and financial integration by fostering closer linkages through cross-border movements within the ASEAN securities markets. ${ }^{2}$ In the capital market, for example, the FTSE/ ASEAN Index series was recently launched as an indicator to the performance of the five oldest stock markets in ASEAN, i.e., the stock markets of Indonesia, Malaysia, Philippines, Singapore and Thailand. This study includes these five countries, which are also the founding members of ASEAN. The different size and the degree of openness of the five markets offer an interesting case study. The markets of Malaysia and Singapore are relatively larger in size, matching the ratio of market capitalization to GDP of the markets in some industrialized countries, and their degree of financial openness is also much higher (Rillo, 2004) compared to the other three markets. By these measurements, Singapore has the most developed market.

This study also proposes a direct modeling of the economic grouping impact using the international asset pricing model (ICAPM), while accounting for the time-varying nature of asset prices. The contribution of this paper is threefold. First, we show that the international asset pricing model, to which an economic grouping factor is added, has a higher explanatory power than the conventional ICAPM models. Second, a dynamic analysis adopted in this study indicates that the pricing mechanism in a more developed market within the economic group is far more stable. Lastly, this study offers new evidence that the stock markets of ASEAN have a higher tendency to converge within the economic group than to the world market.

The rest of this paper is organized as follows. Section 2 introduces the modified ICAPM model to study economic grouping framework and the methodology for analysis. Section 3 reports the results and Section 4 concludes the paper.

\section{Models and Methodology}

The ICAPM offers a theoretical framework for the pricing of risky assets in a fully integrated world of financial markets. The conditional expected return of a national equity market is exposed to the movement in returns of the world portfolio given by the following process:

$$
E\left(R_{i t} \mid \square_{t-1}\right)-R_{F t}=\delta_{W t} \operatorname{cov}\left(R_{i t}, R_{W t} \mid \square_{t-1}\right) ; \forall i
$$

where $R_{i t}$ and $R_{w t}$ represent the returns of market- $i$ and global portfolio, respectively, is the world risk-free rate, and all expectations are taken with respect to the information set available at time $t-1, ; \Omega_{t-1}$. This version of one-

${ }^{2}$ http://www.ASEAN.org. 
factor ICAPM assumes that the expected excess return of an individual market above an international risk-free rate is proportional to the country specific and non-diversifiable risk in the world market. The global portfolio return is the only source of systematic risk that affects the return of each individual market in model (1).

Some researchers including Errunza and Losq (1985), Campbell and Hamao (1992), Davidson et al. (2003) and Bekaert et al. (2005) have added the returns of dominant markets in the region into the ICAPM to reflect the regional effects. In this paper, the region refers to Asia. We consider a two-factor model given as:

$$
E\left(R_{i t} \mid \square_{t-1}\right)-R_{F t}=\delta_{R t} \operatorname{Cov}\left(R_{i t}, R_{R t} \mid \square_{t-1}\right)+\delta_{W t} \operatorname{Cov}\left(R_{i t}, R_{W t} \mid \square_{t-1}\right) ; \forall i
$$

where $R_{R t}$ represents the return to a regional portfolio. Apart from exposure to the world market risk, model (2) includes movements in the markets within the region as an additional source of systematic risk that affects the return of each individual market. Although the regional influence is included, the effect due to formation of economic groupings is not considered in the two-factor model. We propose a three-factor model specified as follows:

$$
\begin{aligned}
& E\left(R_{i t} \mid \square_{t-1}\right)-R_{F t}=\delta_{G t} \operatorname{Cov}\left(R_{i t}, R_{G t} \mid \square_{t-1}\right)+\delta_{R t} \operatorname{Cov}\left(R_{i t}, R_{R t} \mid \square_{t-1}\right) \\
& +\delta_{W t} \operatorname{Cov}\left(R_{i t}, R_{W t} \mid \square_{t-1}\right) ; \forall i
\end{aligned}
$$

where $R_{G t}$, the returns to the portfolio of the economic group, provides a new factor to capture the risk exposure to the grouping where country- $i$ is a member.

Following the above discussion, a number of different versions of ICAPM are obtained. For ease of exposition, the conditional expectation operation is dropped. Three versions of one-factor ICAPM model are considered. First is the version that relates to the world market systematic risk that is given by:

$$
E R_{i t}=\alpha_{i}+\beta_{i}^{W} E R_{W t}+\varepsilon_{i t}
$$

where $E R_{i}$ denotes the excess return of market- $i$ and $E R_{W}$ is the global portfolio excess return. The coefficient $\beta_{i}^{W}$ captures the systematic risk of market$i$ due to exposure to the world market, as suggested in model (1). The other two single-factor models account for the exposure to market risk of the region and market risk of the economic grouping. These models are stated as

$$
E R_{i t}=\alpha_{i}+\beta_{i}^{R} E R_{R t}+\varepsilon_{i t}
$$

and

$$
E R_{i t}=\alpha_{i}+\beta_{i}^{G} E R_{G t}+\varepsilon_{i t}
$$

where $E R_{R}$ and $E R_{G}$ represent the excess returns of the regional portfolio and economic grouping portfolio, respectively. Henceforth, we refer to models (4), (5) and (6) as the W-CAPM, R-CAPM and G-CAPM, respectively. 
Three possible specifications of two-factor ICAPM can be formed by including the exposure to market risks of the region and world, economic grouping and world, and, economic grouping and region. The specifications of the two-factor models are as follows:

$$
\begin{aligned}
& E R_{i t}=\alpha_{i}+\beta_{i}^{R} E R_{R t}+\beta_{i}^{W} E R_{W t}+\varepsilon_{i t} \\
& E R_{i t}=\alpha_{i}+\beta_{i}^{G} E R_{G t}+\beta_{i}^{W} E R_{W t}+\varepsilon_{i t} \\
& E R_{i t}=\alpha_{i}+\beta_{i}^{G} E R_{G t}+\beta_{i}^{R} E R_{R t}+\varepsilon_{i t}
\end{aligned}
$$

The models are referred to as RW-CAPM, GW-CAPM and GR-CAPM, respectively in this paper.

The three-factor ICAPM model incorporates the economic grouping, regional and global effects. The model, denoted as GRW-CAPM, is as follows:

$$
E R_{i t}=\alpha_{i}+\beta_{i}^{G} E R_{G t}+\beta_{i}^{R} E R_{R t}+\beta_{i}^{W} E R_{W t}+\varepsilon_{i t}
$$

We conduct a preliminary analysis of models (4) to (10) using OLS estimation in a static framework. The best model is selected based on the Akaike information criterion (AIC) and Schwarz criterion (SC). The parameter stability of the selected model is examined using the CUSUM of squares test (Brown et al., 1975) and the N-step forecast test. Evidence of recursive residuals outside the error bounds determined at the 5 per cent level is taken to indicate parameter or variance instability.

The selected model is then re-estimated using the generalized autoregressive conditional heteroscedasticity (GARCH) model of Bollerslev (1986) to account for temporal dependence in unconditional residuals which can be induced by time-varying volatility. The conditional expectation underlying models (1) to (3) is stated as follows:

$$
\varepsilon_{i t} \mid \square_{t-1} \sim N\left(0, \sigma_{i t}^{2}\right)
$$

where, the conditional variance $\sigma_{i t}^{2}$ follows the specification which can be written as:

$$
\sigma_{i t}^{2}=\omega+\alpha \varepsilon_{i, t-1}^{2}+\beta \sigma_{i, t-1}^{2}
$$

This simple specification of $\operatorname{GARCH}(1,1)$ is found to be generally sufficient for empirical modeling (Engle and $\mathrm{Ng}$, 1993). To account for non-normal conditional residual distribution, we apply the robust consistent variance-covariance estimator suggested by Bollerslev and Wooldridge (1992). A dynamic framework is used whereby the model is estimated using rolling regression method. The window is set at 3 years.

This study is based on monthly data collected for the period January 1988 and November 2004. The Morgan Stanley Capital International (MSCI) Country 
Index is used in the computation of returns of the individual country. The world portfolio is proxied by the MSCI All Country World Index, a free float-adjusted market capitalization index computed from the stock exchanges of 49 leading markets. The MSCI All Country Asia Index based on indices of 12 leading Asian markets is used to compute the returns on the regional portfolio. The return on the portfolio of the ASEAN group for a member country is computed from an equal weighted market index of the remaining four members. The three-month Treasury bill rates of US, downloaded from the website of The Federal Reserve, represent the risk-free rate used in this study.

\section{Results}

Table 1: presents some summary statistics and the correlation matrix of the market returns.

Table 1: Summary Statistics and Correlation Matrix of Returns

\begin{tabular}{lrrrrrrr}
\hline $\begin{array}{l}\text { Summary } \\
\text { Statistics }\end{array}$ & Indonesia & \multicolumn{7}{c}{ Malaysia Philippines } & Singapore & Thailand & Region & World \\
\hline Mean & 0.004 & 0.004 & 0.002 & 0.005 & 0.002 & -0.001 & 0.005 \\
Std. Dev. & 0.149 & 0.093 & 0.098 & 0.073 & 0.121 & 0.063 & 0.042 \\
Skewness & 0.422 & -0.207 & -0.009 & -0.478 & -0.381 & 0.002 & -0.566 \\
Kurtosis & 7.042 & 6.415 & 4.609 & 5.152 & 4.628 & 3.468 & 3.785 \\
Jarque-Bera & 144.206 & 100.091 & 21.910 & 46.912 & 27.319 & 1.855 & 16.045 \\
(p-value) & $(0.000)$ & $(0.000)$ & $(0.000)$ & $(0.000)$ & $(0.000)$ & $(0.396)$ & $(0.000)$ \\
No. of & 203 & 203 & 203 & 203 & 203 & 203 & 203 \\
Observations & & & & & & & \\
Correlation & Indonesia & Malaysia Philippines & Singapore & Thailand & Region & World \\
\hline Indonesia & 1 & & & & & & \\
Malaysia & 0.479 & 1 & & & & & \\
Philippines & 0.500 & 0.549 & 1 & & & & \\
Singapore & 0.505 & 0.658 & 0.617 & 1 & & & \\
Thailand & 0.466 & 0.566 & 0.634 & 0.655 & 1 & & \\
Region & 0.231 & 0.391 & 0.348 & 0.528 & 0.438 & & \\
World & 0.261 & 0.424 & 0.409 & 0.622 & 0.467 & 0.785 & \\
\hline & & & & & & & \\
\hline
\end{tabular}

Note: Std. Dev. - standard deviation.

With the exception of the regional returns, the Jarque-Bera test rejects the normality assumption of the unconditional distribution of all the series. The returns of the Indonesian market have the highest standard deviation while the return variability of the Singapore market is the lowest. The ASEAN markets 
experienced more volatile movements than the global market. All correlation coefficients are positive, indicating co-movements of the same direction. Malaysia and Singapore are the most closely related markets compared to other markets. The Indonesian market has the lowest correlation not only with its ASEAN counterparts, but also with the regional and world markets. Being the most developed and liberalized stock market, the Singapore market has the highest correlation with the regional and world markets.

\subsection{Static Estimation}

The results of the OLS estimation of the W-, R- G-, RW-, GW-, GR- and GRWCAPM models stated in equations (4) to (10) are reported in Table 2.

Note that the economic grouping factor is significant at $1 \%$ in all the models across all five countries. The inclusion of the economic grouping factor also increases the adjusted- $\mathrm{R}^{2}$, i.e., models with this factor has better explanatory power than models without. The world and regional returns are not always significant in explaining returns of the individual markets. While regional returns are significant in the one-factor R-CAPM model, they are not significant with the incorporation of the global returns in the RW-CAPM model. Similarly, the regional factor is also not significant in the GRW-CAPM model. This suggests that the world market movements have higher influence on the ASEAN stock markets compared to regional dynamics. The economic grouping dynamics have higher impact than the world market movements. Of the GRW-CAPM models, the economic grouping is always significant, but the world factor is only significant in two out of the five models.

The magnitude of the betas suggests that the economic grouping factor has a dominant role in equity pricing of the ASEAN markets. The presence of economic grouping factor reduces the magnitude of the world and regional betas when the one-factor model is extended to the two- and three-factor models. The exposure to systematic risks due to economic grouping is also consistently higher than those due to the regional and world factors. The only exception is Singapore, where exposure to the world factor is higher than the economic grouping factor.

The above findings are further supported by the model selection result. The GW-CAPM model outperforms the other models in four of the markets, while the GR-CAPM setting is the preferred model for Thailand. The results are indicative that inclusion of the economic grouping returns has enhanced the goodness-of-fit and explanatory power of the conventional ICAPM model. The diagnostic results, however, suggest that the OLS estimation suffers from ARCH effects. This problem is taken into account in the next section.

Two tests are conducted to show that the selected models experienced parameter instability. The results of the CUSUM of squares and N-step forecast tests are illustrated in Figure 1. Both the tests consistently show that the parameters in the selected models are not stable over time in all but the Philippine market. Some instability occurred in the early 1990s, but instability is most predominantly around the period of the 1997 financial crisis. To deal with this problem, the rolling window estimation is conducted. 
Table 2: ICAPM Models-Static Estimations

\begin{tabular}{|c|c|c|c|c|c|c|c|c|c|c|c|c|}
\hline Model & Constant & Group & Region & World & $\mathrm{Q}(12)$ & $\mathrm{Q}^{2}(12)$ & Normality & ARCH LM & $\mathrm{F}$ & $\operatorname{Adj} R^{2}$ & AIC & $\mathrm{SC}$ \\
\hline \multicolumn{13}{|l|}{ Indonesia } \\
\hline \multirow[t]{2}{*}{ W-CAPM } & 0.001 & & & 0.778 & 24.627 & 32.905 & 180.521 & 4.026 & 31.378 & 0.131 & -1.040 & -1.007 \\
\hline & $(0.889)$ & & & $(0.000)^{* * *}$ & $(0.017) * *$ & $(0.001) * * *$ & $(0.000)^{* * *}$ & $(0.000)^{* * *}$ & $(0.000)^{* * *}$ & & & \\
\hline \multirow[t]{2}{*}{ R-CAPM } & 0.006 & & 0.621 & & 20.557 & 28.920 & 156.448 & 3.627 & 27.277 & 0.115 & -1.022 & -0.989 \\
\hline & $(0.531)$ & & $(0.000) * * *$ & & $(0.057)^{*}$ & $(0.004)^{* * *}$ & $(0.000)^{* * *}$ & $(0.000)^{* * *}$ & $(0.000)^{* * *}$ & & & \\
\hline \multirow[t]{2}{*}{ G-CAPM } & 0.001 & 0.937 & & & 15.509 & 40.940 & 418.920 & 7.695 & 119.114 & 0.369 & -1.360 & -1.327 \\
\hline & $(0.867)$ & $(0.000)^{* * *}$ & & & $(0.215)$ & $(0.000) * * *$ & $(0.000)^{* * *}$ & $(0.000)^{* * *}$ & $(0.000)^{* * *}$ & & & \\
\hline \multirow[t]{2}{*}{ RW-CAPM } & 0.002 & & 0.170 & 0.601 & 23.293 & 31.813 & 181.006 & 3.950 & 15.871 & 0.128 & -1.032 & -0.983 \\
\hline & $(0.807)$ & & $(0.557)$ & $(0.043)^{* *}$ & $(0.025)^{* *}$ & $(0.001)^{* * *}$ & $(0.000)^{* * *}$ & $(0.000)^{* * *}$ & $(0.000)^{* * *}$ & & & \\
\hline \multirow[t]{2}{*}{ GW-CAPM } & 0.004 & 1.147 & & -0.391 & 13.693 & 41.485 & 374.164 & 8.270 & 63.281 & $\underline{0.381}$ & $\underline{-1.375}$ & -1.326 \\
\hline & $(0.679)$ & $(0.000) * * *$ & & $(0.027)^{* *}$ & $(0.321)$ & $(0.000)^{* * *}$ & $(0.000)^{* * *}$ & $(0.000)^{* * *}$ & $(0.000)^{* * *}$ & & & \\
\hline \multirow[t]{2}{*}{ GR-CAPM } & 0.001 & 1.095 & -0.266 & & 13.072 & 41.079 & 375.583 & 8.198 & 62.218 & 0.377 & -1.369 & -1.320 \\
\hline & $(0.881)$ & $(0.000)^{* * *}$ & $(0.050)^{* *}$ & & $(0.364)$ & $(0.000)^{* * *}$ & $(0.000)^{* * *}$ & $(0.000)^{* * *}$ & $(0.000)^{* * *}$ & & & \\
\hline \multirow[t]{2}{*}{ GRW-CAPM } & 0.003 & 1.152 & -0.070 & -0.323 & 13.359 & 41.442 & 370.343 & 8.314 & 42.034 & 0.379 & -1.366 & -1.300 \\
\hline & $(0.708)$ & $(0.000)^{* * *}$ & $(0.749)$ & $(0.248)$ & $(0.343)$ & $(0.000) * * *$ & $(0.000)^{* * *}$ & $(0.000)^{* * *}$ & $(0.000)^{* * *}$ & & & \\
\hline
\end{tabular}


ح Table 2: ICAPM Models-Static Estimations (continued)

\begin{tabular}{|c|c|c|c|c|c|c|c|c|c|c|c|c|}
\hline \multicolumn{13}{|l|}{ Malaysia } \\
\hline W-CAPM & 0.000 & & & 0.876 & 31.640 & 167.200 & 59.203 & 7.642 & 115.337 & 0.361 & -2.105 & -2.072 \\
\hline & $(0.030)^{* *}$ & & & $(11.795)$ & $(0.002)^{* * *}$ & $(0.000) * * *$ & $(0.000)^{* * *}$ & $(0.000)^{* * *}$ & $(0.000)^{* * *}$ & & & \\
\hline R-CAPM & 0.006 & & 0.705 & & 21.594 & 113.650 & 32.372 & 6.126 & 98.538 & 0.326 & -2.051 & -2.018 \\
\hline & $(0.344)$ & & $(0.000)^{* * *}$ & & $(0.042)^{* *}$ & $(0.000) * * *$ & $(0.000) * * *$ & $(0.000)^{* * *}$ & $(0.000)^{* * *}$ & & & \\
\hline G-CAPM & 0.003 & 0.747 & & & 12.129 & 82.995 & 16.221 & 4.714 & 263.557 & 0.565 & -2.489 & -2.457 \\
\hline & $(0.596)$ & $(0.000)^{* * *}$ & & & $(0.435)$ & $(0.000) * * *$ & $(0.000) * * *$ & $(0.000) * * *$ & $(0.000)^{* * *}$ & & & \\
\hline RW-CAPM & 0.002 & & 0.222 & 0.644 & 26.060 & 153.060 & 53.899 & 7.384 & 59.162 & 0.365 & -2.106 & -2.058 \\
\hline & $(0.798)$ & & $(0.120)$ & $(0.000) * * *$ & $(0.011)^{* *}$ & $(0.000) * * *$ & $(0.000)^{* * *}$ & $(0.000)^{* * *}$ & $(0.000)^{* * *}$ & & & \\
\hline GW-CAPM & 0.001 & 0.632 & & 0.247 & 14.478 & 123.060 & 20.584 & 6.333 & 139.751 & $\underline{0.579}$ & $\underline{-2.516}$ & -2.467 \\
\hline & $(0.813)$ & $(0.000)^{* * *}$ & & $(0.017)^{* *}$ & $(0.271)$ & $(0.000)^{* * *}$ & $(0.000)^{* * *}$ & $(0.000)^{* * *}$ & $(0.000)^{* * *}$ & & & \\
\hline GR-CAPM & 0.003 & 0.646 & 0.195 & & 16.734 & 115.290 & 21.925 & 5.977 & 139.419 & 0.578 & -2.515 & -2.466 \\
\hline & $(0.590)$ & $(0.000) * * *$ & $(0.008) * * *$ & & $(0.160)$ & $(0.000)^{* * *}$ & $(0.000) * * *$ & $(0.000)^{* * *}$ & $(0.000) * * *$ & & & \\
\hline GRW-CAPM & 0.002 & 0.627 & 0.100 & 0.148 & 15.779 & 123.570 & 21.892 & 6.318 & 93.247 & 0.578 & -2.510 & -2.444 \\
\hline & $(0.722)$ & $(0.000)^{* * *}$ & $(0.402)$ & $(0.382)$ & $(0.202)$ & $(0.000)^{* * *}$ & $(0.000) * * *$ & $(0.000)^{* * *}$ & $(0.000)^{* * *}$ & & & \\
\hline
\end{tabular}


Table 2: ICAPM Models-Static Estimations (continued)

\begin{tabular}{|c|c|c|c|c|c|c|c|c|c|c|c|c|}
\hline Model & Constant & Group & Region & World & $\mathrm{Q}(12)$ & $\mathrm{Q}^{2}(12)$ & Normality & ARCH LM & $\mathrm{F}$ & $\operatorname{Adj} R^{2}$ & AIC & $\mathrm{SC}$ \\
\hline \multicolumn{13}{|l|}{ Philippines } \\
\hline \multirow[t]{2}{*}{ W-CAPM } & -0.004 & & & 1.040 & 17.229 & 13.430 & 2.440 & 1.062 & 144.366 & 0.415 & -1.986 & -1.953 \\
\hline & $(0.550)$ & & & $(0.000)^{* * *}$ & $(0.141)$ & $(0.339)$ & $(0.295)$ & $(0.395)$ & $(0.000)^{* * *}$ & & & \\
\hline \multirow[t]{2}{*}{ R-CAPM } & 0.003 & & 0.799 & & 13.036 & 6.769 & 0.256 & 0.493 & 105.099 & 0.340 & -1.865 & -1.832 \\
\hline & $(0.634)$ & & $(0.000)^{* * *}$ & & $(0.366)$ & $(0.873)$ & $(0.880)$ & $(0.917)$ & $(0.000)^{* * *}$ & & & \\
\hline \multirow[t]{2}{*}{ G-CAPM } & -0.001 & 0.894 & & & 10.113 & 25.129 & 0.121 & 2.270 & 329.290 & 0.619 & -2.415 & -2.382 \\
\hline & $(0.791)$ & $(0.000)^{* * *}$ & & & $(0.606)$ & $(0.014)^{* *}$ & $(0.941)$ & $(0.011)^{* *}$ & $(0.000)^{* * *}$ & & & \\
\hline \multirow[t]{2}{*}{ RW-CAPM } & -0.003 & & 0.087 & 0.949 & 16.619 & 12.645 & 1.704 & 0.986 & 72.086 & 0.413 & -1.977 & -1.928 \\
\hline & $(0.611)$ & & $(0.600)$ & $(0.000) * * *$ & $(0.164)$ & $(0.395)$ & $(0.427)$ & $(0.464)$ & $(0.000) * * *$ & & & \\
\hline \multirow[t]{2}{*}{ GW-CAPM } & -0.003 & 0.732 & & 0.342 & 10.408 & 27.965 & 1.653 & 2.378 & 182.228 & $\underline{0.642}$ & -2.472 & -2.423 \\
\hline & $(0.497)$ & $(0.000)^{* * *}$ & & $(0.000) * * *$ & $(0.580)$ & $(0.006)^{* * *}$ & $(0.437)$ & $(0.007)^{* * *}$ & $(0.000)^{* * *}$ & & & \\
\hline \multirow[t]{2}{*}{ GR-CAPM } & -0.001 & 0.792 & 0.193 & & 9.841 & 22.971 & 0.210 & 1.910 & 172.397 & 0.629 & -2.437 & -2.388 \\
\hline & $(0.803)$ & $(0.000)^{* * *}$ & $(0.006)^{* * *}$ & & $(0.630)$ & $(0.028)^{* *}$ & $(0.900)$ & $(0.036)^{* *}$ & $(0.000)^{* * *}$ & & & \\
\hline \multirow[t]{2}{*}{ GRW-CAPM } & -0.004 & 0.737 & -0.081 & 0.422 & 10.547 & 28.879 & 2.156 & 2.497 & 121.282 & 0.641 & -2.464 & -2.399 \\
\hline & $(0.432)$ & $(0.000)^{* * *}$ & $(0.531)$ & $(0.018)^{* *}$ & $(0.568)$ & $(0.004)^{* * *}$ & $(0.340)$ & $(0.005)^{* * *}$ & $(0.000)^{* * *}$ & & & \\
\hline
\end{tabular}


స Table 2: ICAPM Models-Static Estimations (continued)

\begin{tabular}{|c|c|c|c|c|c|c|c|c|c|c|c|c|}
\hline \multicolumn{13}{|l|}{ Singapore } \\
\hline W-CAPM & 0.001 & & & 0.948 & 12.226 & 101.370 & 36.305 & 6.307 & 293.565 & 0.592 & -2.882 & -2.849 \\
\hline & $(0.849)$ & & & $(0.000)^{* * *}$ & $(0.428)$ & $(0.000)^{* * *}$ & $(0.000)^{* * *}$ & $(0.000)^{* * *}$ & $(0.000)^{* * *}$ & & & \\
\hline R-CAPM & 0.007 & & 0.741 & & 12.061 & 59.878 & 6.159 & 5.096 & 204.807 & 0.502 & -2.684 & -2.652 \\
\hline & $(0.119)$ & & $(0.000)^{* * *}$ & & $(0.441)$ & $(0.000)^{* * *}$ & $(0.046) * *$ & $(0.000)^{* * *}$ & $(0.000)^{* * *}$ & & & \\
\hline G-CAPM & 0.005 & 0.675 & & & 15.281 & 23.029 & 41.912 & 1.881 & 411.017 & 0.670 & -3.095 & -3.063 \\
\hline & $(0.205)$ & $(0.000)^{* * *}$ & & & $(0.226)$ & $(0.027)^{* *}$ & $(0.000)^{* * *}$ & $(0.039) * *$ & $(0.000)^{* * *}$ & & & \\
\hline RW-CAPM & 0.002 & & 0.138 & 0.804 & 10.676 & 93.288 & 32.790 & 6.304 & 148.372 & 0.593 & -2.882 & -2.833 \\
\hline & $(0.688)$ & & $(0.164)$ & $(0.000)^{* * *}$ & $(0.557)$ & $(0.000)^{* * *}$ & $(0.000)^{* * *}$ & $(0.000)^{* * *}$ & $(0.000)^{* * *}$ & & & \\
\hline GW-CAPM & 0.002 & 0.454 & & 0.503 & 16.363 & 47.638 & 45.667 & 2.775 & 329.416 & $\underline{0.765}$ & -3.429 & -3.380 \\
\hline & $(0.613)$ & $(0.000)^{* * *}$ & & $(0.000)^{* * *}$ & $(0.175)$ & $(0.000)^{* * *}$ & $(0.000)^{* * *}$ & $(0.002)^{* * *}$ & $(0.000)^{* * *}$ & & & \\
\hline GR-CAPM & 0.005 & 0.507 & 0.345 & & 25.006 & 40.655 & 76.921 & 2.978 & 283.661 & 0.737 & -3.316 & -3.268 \\
\hline & $(0.167)$ & $(0.000)^{* * *}$ & $(0.000)^{* * *}$ & & $(0.015)^{* *}$ & $(0.000)^{* * *}$ & $(0.000)^{* * *}$ & $(0.001)^{* * *}$ & $(0.000)^{* * *}$ & & & \\
\hline GRW-CAPM & 0.002 & 0.452 & 0.040 & 0.464 & 17.547 & 47.577 & 49.807 & 2.828 & 218.899 & 0.764 & -3.421 & -3.355 \\
\hline & $(0.557)$ & $(0.000)^{* * *}$ & $(0.615)$ & $(0.000) * * *$ & $(0.130)$ & $(0.000) * * *$ & $(0.000)^{* * *}$ & $(0.001)^{* * *}$ & $(0.000)^{* * *}$ & & & \\
\hline
\end{tabular}


Table 2: ICAPM Models-Static Estimations (continued)

\begin{tabular}{|c|c|c|c|c|c|c|c|c|c|c|c|c|}
\hline Model & Constant & Group & Region & World & $\mathrm{Q}(12)$ & $\mathrm{Q}^{2}(12)$ & Normality & ARCH LM & $\mathrm{F}$ & $\operatorname{Adj} R^{2}$ & AIC & $\mathrm{SC}$ \\
\hline \multicolumn{13}{|l|}{ Thailand } \\
\hline \multirow[t]{2}{*}{ W-CAPM } & -0.005 & & & 1.216 & 30.032 & 103.230 & 10.534 & 4.376 & 135.878 & 0.400 & -1.612 & -1.580 \\
\hline & $(0.538)$ & & & $(0.000)^{* * *}$ & $(0.003)^{* * *}$ & $(0.000)^{* * *}$ & $(0.005)^{* * *}$ & $(0.000)^{* * *}$ & $(0.000)^{* * *}$ & & & \\
\hline \multirow[t]{2}{*}{ R-CAPM } & 0.003 & & 0.996 & & 18.874 & 61.432 & 1.676 & 3.271 & 121.346 & 0.373 & -1.568 & -1.536 \\
\hline & $(0.694)$ & & $(0.000)^{* * *}$ & & $(0.092)^{*}$ & $(0.000) * * *$ & $(0.432)$ & $(0.000)^{* * *}$ & $(0.000)^{* * *}$ & & & \\
\hline \multirow[t]{2}{*}{ G-CAPM } & -0.002 & 1.101 & & & 16.263 & 36.122 & 297.860 & 3.102 & 309.758 & 0.605 & -2.029 & -1.996 \\
\hline & $(0.721)$ & $(0.000)^{* * *}$ & & & $(0.180)$ & $(0.000)^{* * *}$ & $(0.000)^{* * *}$ & $(0.001)^{* * *}$ & $(0.000)^{* * *}$ & & & \\
\hline \multirow[t]{2}{*}{ RW-CAPM } & -0.002 & & 0.386 & 0.814 & 24.646 & 86.510 & 5.621 & 4.064 & 71.119 & 0.410 & -1.623 & -1.574 \\
\hline & $(0.751)$ & & $(0.063)^{*}$ & $(0.000)^{* * *}$ & $(0.017)^{* *}$ & $(0.000)^{* * *}$ & $(0.060)^{* *}$ & $(0.000)^{* * *}$ & $(0.000)^{* * *}$ & & & \\
\hline \multirow[t]{2}{*}{ GW-CAPM } & -0.004 & 0.908 & & 0.390 & 15.154 & 41.754 & 148.087 & 3.378 & 169.663 & 0.625 & -2.078 & -2.029 \\
\hline & $(0.450)$ & $(0.000)^{* * *}$ & & $(0.009)^{* * *}$ & $(0.233)$ & $(0.000)^{* * *}$ & $(0.000)^{* * *}$ & $(0.000)^{* * *}$ & $(0.000)^{* * *}$ & & & \\
\hline \multirow[t]{2}{*}{ GR-CAPM } & -0.002 & 0.917 & 0.339 & & 14.570 & 44.631 & 144.553 & 3.879 & 172.567 & $\underline{0.629}$ & -2.089 & $\underline{-2.040}$ \\
\hline & $(0.734)$ & $(0.000) * * *$ & $(0.005)^{* * *}$ & & $(0.266)$ & $(0.000)^{* * *}$ & $(0.000)^{* * *}$ & $(0.000)^{* * *}$ & $(0.000)^{* * *}$ & & & \\
\hline GRW-CAPM & $\begin{array}{l}-0.003 \\
(0.617)\end{array}$ & $\begin{array}{l}0.896 \\
(0.000)^{* * *}\end{array}$ & $\begin{array}{l}0.248 \\
(0.116)\end{array}$ & $\begin{array}{l}0.142 \\
(0.471)\end{array}$ & $\begin{array}{l}14.816 \\
(0.252)\end{array}$ & $\begin{array}{l}44.698 \\
(0.000)^{* * *}\end{array}$ & $\begin{array}{l}134.620 \\
(0.000)^{* * *}\end{array}$ & $\begin{array}{l}3.799 \\
(0.000) * * *\end{array}$ & $\begin{array}{l}114.994 \\
(0.000)^{* * *}\end{array}$ & 0.629 & -2.082 & -2.017 \\
\hline
\end{tabular}

Note: Figures in the parentheses are the p-values. * denotes significance at the 0.10 level; $* *$ denotes significance at the 0.05 level; and $* * *$ denotes significance at the 0.01 level. The coefficient significance test is based on the White (1980) heteroskedasticity consistent covariance estimates. AIC and SC denotes the Akaike and Schwarz information criteria. The underlined values show the model chosen using adjusted (Adj) R², AIC and SC. Q(12) and $\mathrm{Q}^{2}(12)$ are the LM tests for serial dependence in the residuals and squared residuals at 12 lags, respectively. Normality is the Jacque-Bera test for normality. ARCH LM is the test for presence of ARCH effects at 12 lags. F indicates the overall test for model significance. 
Figure 1: Tests for Parameter Instability

\section{CUSUM of Squares Test}

\section{N-step Forecast Test}

Indonesia
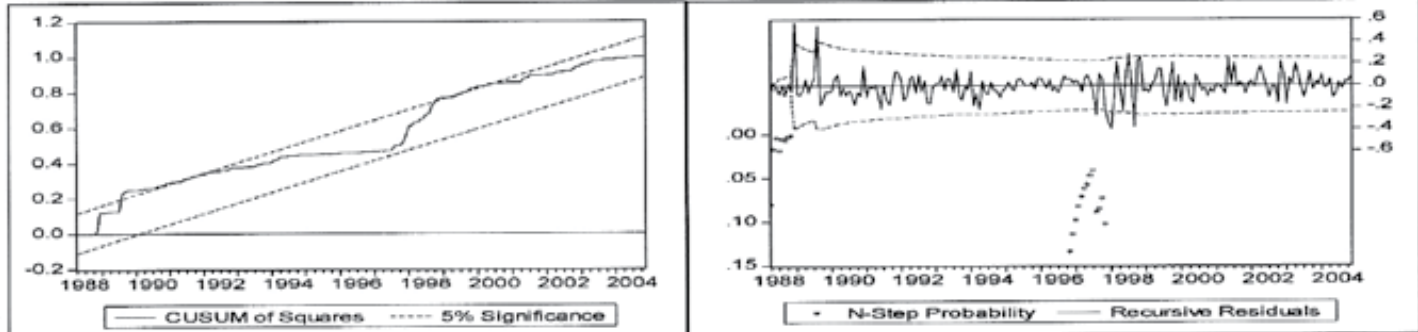

Malaysia
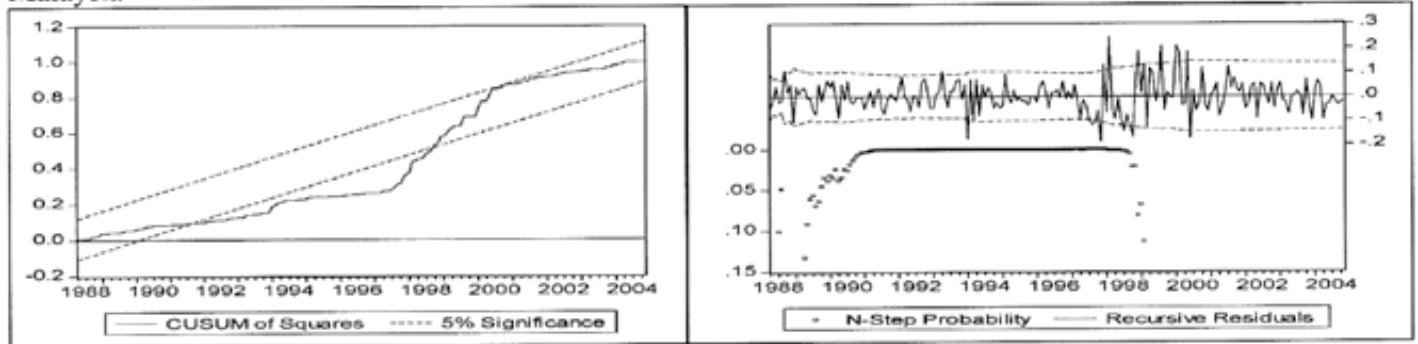

Philippines
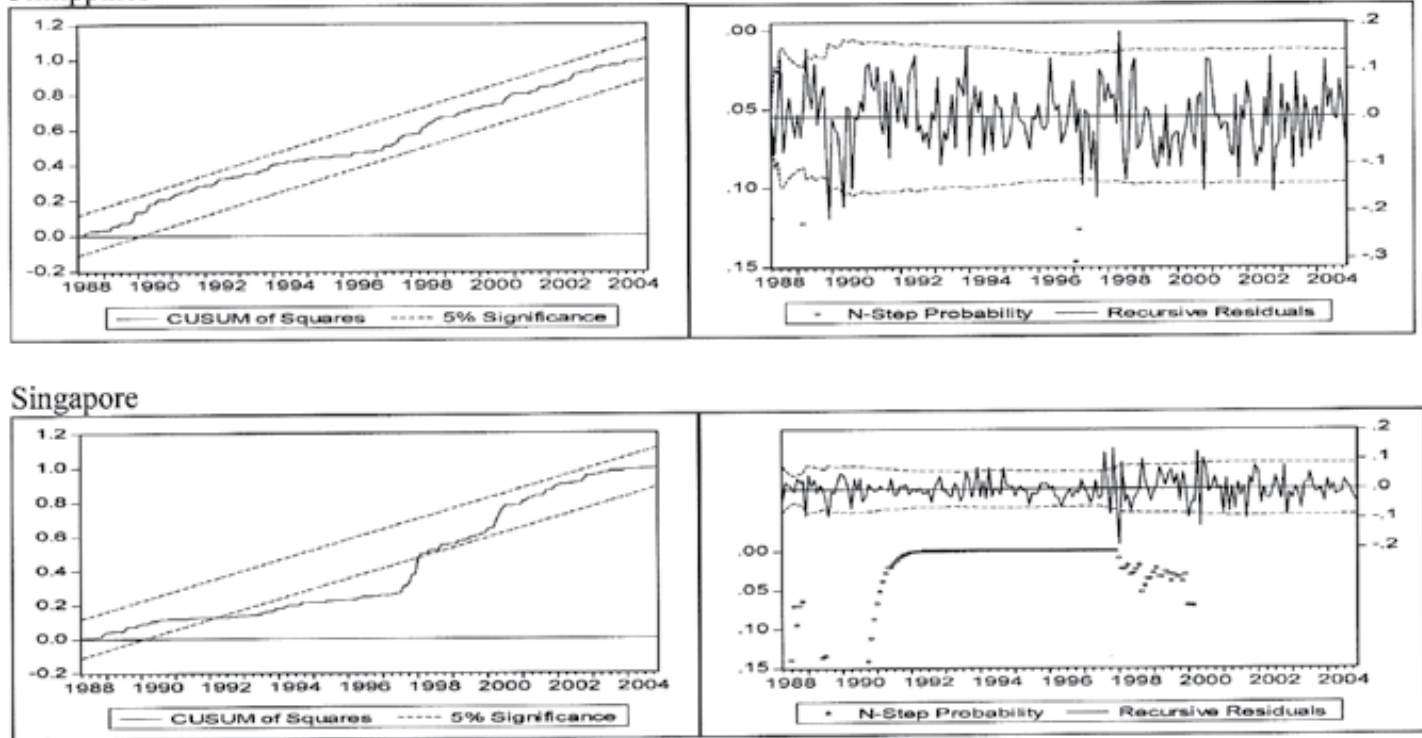

Thailand
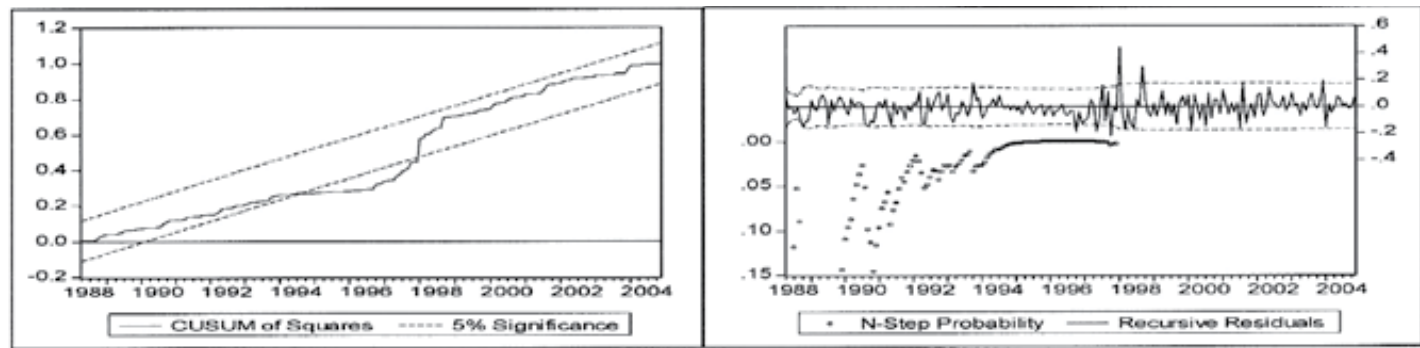
Table 3: Rolling Regression Estimates of the GW-CAPM Model for Indonesia

\begin{tabular}{|c|c|c|c|c|c|c|c|c|c|c|c|}
\hline & Constant Mean & Group & World & Constant Variance & $\mathrm{ARCH}$ & GARCH & $\mathrm{Q}(12)$ & $\mathrm{Q}^{2}(12)$ & Normality & $\mathrm{ARCH} \mathrm{LM}$ & $\operatorname{Adj~} R^{2}$ \\
\hline $88 / 1-90 / 12$ & $\begin{array}{l}0.012 \\
(0.420)\end{array}$ & $\begin{array}{l}0.422 \\
(0.100) * * *\end{array}$ & $\begin{array}{l}-0.806 \\
(0.011)^{* *}\end{array}$ & $\begin{array}{l}0.001 \\
(0.701)\end{array}$ & $\begin{array}{l}-0.112 \\
(0.572)\end{array}$ & $\begin{array}{l}1.183 \\
(0.000)^{* * *}\end{array}$ & $\begin{array}{l}11.397 \\
(0.495)\end{array}$ & $\begin{array}{l}12.177 \\
(0.432)\end{array}$ & $\begin{array}{l}8.458 \\
(0.015)^{* *}\end{array}$ & $\begin{array}{l}0.335 \\
(0.963)\end{array}$ & 0.156 \\
\hline $89 / 1-91 / 12$ & $\begin{array}{l}-0.013 \\
(0.271)\end{array}$ & $\begin{array}{l}1.416 \\
(0.000) * * *\end{array}$ & $\begin{array}{l}-1.351 \\
(0.033)^{* *}\end{array}$ & $\begin{array}{l}0.007 \\
(0.018) * *\end{array}$ & $\begin{array}{l}0.910 \\
(0.381)\end{array}$ & $\begin{array}{l}-0.047 \\
(0.007) * * *\end{array}$ & $\begin{array}{l}10.782 \\
(0.548)\end{array}$ & $\begin{array}{l}3.082 \\
(0.995)\end{array}$ & $\begin{array}{l}6.805 \\
(0.033) * *\end{array}$ & $\begin{array}{l}0.479 \\
(0.889)\end{array}$ & 0.128 \\
\hline $90 / 1-92 / 12$ & $\begin{array}{l}-0.023 \\
(0.007)^{* * *}\end{array}$ & $\begin{array}{l}1.273 \\
(0.000)^{* * *}\end{array}$ & $\begin{array}{l}-0.724 \\
(0.000)^{* * *}\end{array}$ & $\begin{array}{l}0.001 \\
(0.001)^{* * *}\end{array}$ & $\begin{array}{l}-0.277 \\
(0.208)\end{array}$ & $\begin{array}{l}1.134 \\
(0.000)^{* * *}\end{array}$ & $\begin{array}{l}14.560 \\
(0.266)\end{array}$ & $\begin{array}{l}20.293 \\
(0.062)^{*}\end{array}$ & $\begin{array}{l}2.015 \\
(0.365)\end{array}$ & $\begin{array}{l}0.699 \\
(0.727)\end{array}$ & 0.406 \\
\hline $91 / 1-93 / 12$ & $\begin{array}{l}-0.019 \\
(0.066) *\end{array}$ & $\begin{array}{l}0.666 \\
(0.000) * * *\end{array}$ & $\begin{array}{l}0.203 \\
(0.155)\end{array}$ & $\begin{array}{l}0.000 \\
(0.000) * * *\end{array}$ & $\begin{array}{l}-0.201 \\
(0.348)\end{array}$ & $\begin{array}{l}1.167 \\
(0.000)^{* * *}\end{array}$ & $\begin{array}{l}5.712 \\
(0.930)\end{array}$ & $\begin{array}{l}13.836 \\
(0.311)\end{array}$ & $\begin{array}{l}1.345 \\
(0.510)\end{array}$ & $\begin{array}{l}0.926 \\
(0.554)\end{array}$ & 0.358 \\
\hline $92 / 1-94 / 12$ & $\begin{array}{l}-0.005 \\
(0.637)\end{array}$ & $\begin{array}{l}0.535 \\
(0.000) * * *\end{array}$ & $\begin{array}{l}0.567 \\
(0.005) * * *\end{array}$ & $\begin{array}{l}0.002 \\
(0.699)\end{array}$ & $\begin{array}{l}-0.027 \\
(0.800)\end{array}$ & $\begin{array}{l}0.531 \\
(0.671)\end{array}$ & $\begin{array}{l}13.250 \\
(0.351)\end{array}$ & $\begin{array}{l}13.024 \\
(0.367)\end{array}$ & $\begin{array}{l}1.520 \\
(0.468)\end{array}$ & $\begin{array}{l}0.901 \\
(0.572)\end{array}$ & 0.567 \\
\hline $93 / 1-95 / 12$ & $\begin{array}{l}0.003 \\
(0.770)\end{array}$ & $\begin{array}{l}0.907 \\
(0.000) * * *\end{array}$ & $\begin{array}{l}0.169 \\
(0.402)\end{array}$ & $\begin{array}{l}0.000 \\
(0.639)\end{array}$ & $\begin{array}{l}0.333 \\
(0.038)^{* *}\end{array}$ & $\begin{array}{l}0.595 \\
(0.087)^{*}\end{array}$ & $\begin{array}{l}14.430 \\
(0.274)\end{array}$ & $\begin{array}{l}17.321 \\
(0.138)\end{array}$ & $\begin{array}{l}0.303 \\
(0.859)\end{array}$ & $\begin{array}{l}0.912 \\
(0.564)\end{array}$ & 0.516 \\
\hline $94 / 1-96 / 12$ & $\begin{array}{l}0.008 \\
(0.142)\end{array}$ & $\begin{array}{l}1.100 \\
(0.000)^{* * *}\end{array}$ & $\begin{array}{l}0.134 \\
(0.345)\end{array}$ & $\begin{array}{l}0.000 \\
(0.445)\end{array}$ & $\begin{array}{l}-0.166 \\
(0.481)\end{array}$ & $\begin{array}{l}1.070 \\
(0.006)^{* * *}\end{array}$ & $\begin{array}{l}6.654 \\
(0.880)\end{array}$ & $\begin{array}{l}7.642 \\
(0.812)\end{array}$ & $\begin{array}{l}1.987 \\
(0.370)\end{array}$ & $\begin{array}{l}0.883 \\
(0.585)\end{array}$ & 0.657 \\
\hline $95 / 1-97 / 12$ & $\begin{array}{l}0.021 \\
(0.000) * * *\end{array}$ & $\begin{array}{l}1.262 \\
(0.000) * * *\end{array}$ & $\begin{array}{l}-0.147 \\
(0.390)\end{array}$ & $\begin{array}{l}0.000 \\
(0.120)\end{array}$ & $\begin{array}{l}1.286 \\
(0.002) * * *\end{array}$ & $\begin{array}{l}0.056 \\
(0.558)\end{array}$ & $\begin{array}{l}11.733 \\
(0.467)\end{array}$ & $\begin{array}{l}7.999 \\
(0.785)\end{array}$ & $\begin{array}{l}1.965 \\
(0.374)\end{array}$ & $\begin{array}{l}0.965 \\
(0.527)\end{array}$ & 0.626 \\
\hline $96 / 1-98 / 12$ & $\begin{array}{l}0.014 \\
(0.070)^{*}\end{array}$ & $\begin{array}{l}1.052 \\
(0.000) * * *\end{array}$ & $\begin{array}{l}0.673 \\
(0.001)^{* * *}\end{array}$ & $\begin{array}{l}0.000 \\
(0.835)\end{array}$ & $\begin{array}{l}0.514 \\
(0.112)\end{array}$ & $\begin{array}{l}0.752 \\
(0.001)^{* * *}\end{array}$ & $\begin{array}{l}12.593 \\
(0.399)\end{array}$ & $\begin{array}{l}7.764 \\
(0.803)\end{array}$ & $\begin{array}{l}1.195 \\
(0.550)\end{array}$ & $\begin{array}{l}0.367 \\
(0.951)\end{array}$ & 0.244 \\
\hline $97 / 1-99 / 12$ & $\begin{array}{l}0.019 \\
(0.442)\end{array}$ & $\begin{array}{l}1.358 \\
(0.000)^{* * *}\end{array}$ & $\begin{array}{l}-0.137 \\
(0.756)\end{array}$ & $\begin{array}{l}0.002 \\
(0.408)\end{array}$ & $\begin{array}{l}0.473 \\
(0.032)^{* *}\end{array}$ & $\begin{array}{l}0.594 \\
(0.000)^{* * *}\end{array}$ & $\begin{array}{l}18.482 \\
(0.102)\end{array}$ & $\begin{array}{l}17.371 \\
(0.136)\end{array}$ & $\begin{array}{l}1.015 \\
(0.602)\end{array}$ & $\begin{array}{l}0.772 \\
(0.669)\end{array}$ & 0.350 \\
\hline $98 / 1-00 / 12$ & $\begin{array}{l}-0.039 \\
(0.001)^{* * *}\end{array}$ & $\begin{array}{l}1.255 \\
(0.000) * * *\end{array}$ & $\begin{array}{l}-0.633 \\
(0.008) * * *\end{array}$ & $\begin{array}{l}-0.001 \\
(0.000)^{* * *}\end{array}$ & $\begin{array}{l}-0.082 \\
(0.634)\end{array}$ & $\begin{array}{l}1.048 \\
(0.000)^{* * *}\end{array}$ & $\begin{array}{l}13.353 \\
(0.344)\end{array}$ & $\begin{array}{l}14.038 \\
(0.298)\end{array}$ & $\begin{array}{l}1.795 \\
(0.408)\end{array}$ & $\begin{array}{l}1.084 \\
(0.450)\end{array}$ & 0.318 \\
\hline $99 / 1-01 / 12$ & $\begin{array}{l}-0.007 \\
(0.655)\end{array}$ & $\begin{array}{l}1.459 \\
(0.000) * * *\end{array}$ & $\begin{array}{l}-0.553 \\
(0.006) * * *\end{array}$ & $\begin{array}{l}0.002 \\
(0.416)\end{array}$ & $\begin{array}{l}-0.083 \\
(0.392)\end{array}$ & $\begin{array}{l}0.853 \\
(0.005)^{* * *}\end{array}$ & $\begin{array}{l}6.882 \\
(0.865)\end{array}$ & $\begin{array}{l}6.210 \\
(0.905)\end{array}$ & $\begin{array}{l}1.929 \\
(0.381)\end{array}$ & $\begin{array}{l}0.532 \\
(0.853)\end{array}$ & 0.532 \\
\hline $00 / 1-02 / 12$ & $\begin{array}{l}0.009 \\
(0.512)\end{array}$ & $\begin{array}{l}1.077 \\
(0.000)^{* * *}\end{array}$ & $\begin{array}{l}-0.318 \\
(0.012)^{* *}\end{array}$ & $\begin{array}{l}0.002 \\
(0.318)\end{array}$ & $\begin{array}{l}-0.239 \\
(0.052)^{*}\end{array}$ & $\begin{array}{l}1.057 \\
(0.000)^{* * *}\end{array}$ & $\begin{array}{l}12.274 \\
(0.424)\end{array}$ & $\begin{array}{l}5.939 \\
(0.919)\end{array}$ & $\begin{array}{l}2.129 \\
(0.345)\end{array}$ & $\begin{array}{l}0.395 \\
(0.937)\end{array}$ & 0.395 \\
\hline 01/1-03/12 & $\begin{array}{l}0.020 \\
(0.042) * *\end{array}$ & $\begin{array}{l}0.802 \\
(0.000) * * *\end{array}$ & $\begin{array}{l}-0.177 \\
(0.033) * *\end{array}$ & $\begin{array}{l}0.001 \\
(0.000) * * *\end{array}$ & $\begin{array}{l}-0.259 \\
(0.027)^{* *}\end{array}$ & $\begin{array}{l}1.131 \\
(0.000) * * *\end{array}$ & $\begin{array}{l}4.433 \\
(0.974)\end{array}$ & $\begin{array}{l}7.066 \\
(0.853)\end{array}$ & $\begin{array}{l}0.890 \\
(0.641)\end{array}$ & $\begin{array}{l}0.411 \\
(0.929)\end{array}$ & 0.154 \\
\hline $02 / 1-04 / 11$ & $\begin{array}{l}0.022 \\
(0.156)\end{array}$ & $\begin{array}{l}0.982 \\
(0.011)^{* *}\end{array}$ & $\begin{array}{l}-0.008 \\
(0.987)\end{array}$ & $\begin{array}{l}0.002 \\
(0.801)\end{array}$ & $\begin{array}{l}0.036 \\
(0.744)\end{array}$ & $\begin{array}{l}0.702 \\
(0.500)\end{array}$ & $\begin{array}{l}7.179 \\
(0.846)\end{array}$ & $\begin{array}{l}22.320 \\
(0.034)^{* *}\end{array}$ & $\begin{array}{l}1.574 \\
(0.455)\end{array}$ & $\begin{array}{l}2.747 \\
(0.060)^{*}\end{array}$ & 0.394 \\
\hline Full Sample & $\begin{array}{l}0.004 \\
(0.524) \\
\end{array}$ & $\begin{array}{l}1.232 \\
(0.000) * * *\end{array}$ & $\begin{array}{l}-0.258 \\
(0.094)^{*} \\
\end{array}$ & $\begin{array}{l}0.000 \\
(0.500) \\
\end{array}$ & $\begin{array}{l}0.306 \\
(0.027)^{* *}\end{array}$ & $\begin{array}{l}0.763 \\
(0.000) * * * \\
\end{array}$ & $\begin{array}{l}8.348 \\
(0.757) \\
\end{array}$ & $\begin{array}{l}18.773 \\
(0.094)^{*} \\
\end{array}$ & $\begin{array}{l}356.015 \\
(0.000)^{* * *}\end{array}$ & $\begin{array}{l}7.839 \\
(0.000) * * *\end{array}$ & 0.359 \\
\hline
\end{tabular}


Table 4: Rolling Regression Estimates of the GW-CAPM Model for Malaysia

\begin{tabular}{|c|c|c|c|c|c|c|c|c|c|c|c|}
\hline & Constant Mean & Group & World & Constant Variance & $\mathrm{ARCH}$ & GARCH & $\mathrm{Q}(12)$ & $\mathrm{Q}^{2}(12)$ & Normality & ARCH LM & Adi $R^{2}$ \\
\hline $88 / 1-90 / 12$ & $\begin{array}{l}0.001 \\
(0.894)\end{array}$ & $\begin{array}{l}0.364 \\
(0.000) * * *\end{array}$ & $\begin{array}{l}0.827 \\
(0.000) * * *\end{array}$ & $\begin{array}{l}0.000 \\
(0.000) * * *\end{array}$ & $\begin{array}{l}-0.219 \\
(0.000) * * *\end{array}$ & $\begin{array}{l}1.124 \\
(0.000) * * *\end{array}$ & $\begin{array}{l}11.312 \\
(0.502)\end{array}$ & $\begin{array}{l}6.797 \\
(0.871)\end{array}$ & $\begin{array}{l}0.603 \\
(0.740)\end{array}$ & $\begin{array}{l}0.631 \\
(0.780)\end{array}$ & 0.619 \\
\hline $89 / 1-91 / 12$ & $\begin{array}{l}0.002 \\
(0.706)\end{array}$ & $\begin{array}{l}0.440 \\
(0.000) * * *\end{array}$ & $\begin{array}{l}0.573 \\
(0.001) * * *\end{array}$ & $\begin{array}{l}0.000 \\
(0.528)\end{array}$ & $\begin{array}{l}0.216 \\
(0.292)\end{array}$ & $\begin{array}{l}0.544 \\
(0.295)\end{array}$ & $\begin{array}{l}13.432 \\
(0.338)\end{array}$ & $\begin{array}{l}7.770 \\
(0.803)\end{array}$ & $\begin{array}{l}1.067 \\
(0.586)\end{array}$ & $\begin{array}{l}0.210 \\
(0.994)\end{array}$ & 0.709 \\
\hline $90 / 1-92 / 12$ & $\begin{array}{l}0.001 \\
(0.765)\end{array}$ & $\begin{array}{l}0.595 \\
(0.000) * * *\end{array}$ & $\begin{array}{l}0.361 \\
(0.000) * * *\end{array}$ & $\begin{array}{l}0.000 \\
(0.000) * * *\end{array}$ & $\begin{array}{l}-0.223 \\
(0.001)^{* * *}\end{array}$ & $\begin{array}{l}1.149 \\
(0.000) * * *\end{array}$ & $\begin{array}{l}6.748 \\
(0.874)\end{array}$ & $\begin{array}{l}10.178 \\
(0.600)\end{array}$ & $\begin{array}{l}1.335 \\
(0.513)\end{array}$ & $\begin{array}{l}0.981 \\
(0.516)\end{array}$ & 0.771 \\
\hline $91 / 1-93 / 12$ & $\begin{array}{l}0.008 \\
(0.000) * * *\end{array}$ & $\begin{array}{l}0.646 \\
(0.000) * * *\end{array}$ & $\begin{array}{l}0.242 \\
(0.036) * *\end{array}$ & $\begin{array}{l}0.001 \\
(0.000) * * *\end{array}$ & $\begin{array}{l}-0.141 \\
(0.336)\end{array}$ & $\begin{array}{l}0.592 \\
(0.010)^{* *}\end{array}$ & $\begin{array}{l}13.635 \\
(0.325)\end{array}$ & $\begin{array}{l}5.892 \\
(0.921)\end{array}$ & $\begin{array}{l}0.468 \\
(0.792)\end{array}$ & $\begin{array}{l}0.371 \\
(0.949)\end{array}$ & 0.708 \\
\hline $92 / 1-94 / 12$ & $\begin{array}{l}0.000 \\
(0.971)\end{array}$ & $\begin{array}{l}0.809 \\
(0.000) * * *\end{array}$ & $\begin{array}{l}0.106 \\
(0.682)\end{array}$ & $\begin{array}{l}0.001 \\
(0.634)\end{array}$ & $\begin{array}{l}0.071 \\
(0.605)\end{array}$ & $\begin{array}{l}0.485 \\
(0.614)^{* * *}\end{array}$ & $\begin{array}{l}19.398 \\
(0.079)^{*}\end{array}$ & $\begin{array}{l}10.390 \\
(0.582)\end{array}$ & $\begin{array}{l}0.512 \\
(0.774)\end{array}$ & $\begin{array}{l}0.463 \\
(0.899)\end{array}$ & 0.640 \\
\hline $93 / 1-95 / 12$ & $\begin{array}{l}-0.004 \\
(0.259)\end{array}$ & $\begin{array}{l}1.037 \\
(0.000)^{* * *}\end{array}$ & $\begin{array}{l}0.174 \\
(0.136)\end{array}$ & $\begin{array}{l}0.000 \\
(0.128)\end{array}$ & $\begin{array}{l}0.931 \\
(0.002) * * *\end{array}$ & $\begin{array}{l}0.216 \\
(0.049) * *\end{array}$ & $\begin{array}{l}26.146 \\
(0.010)^{* *}\end{array}$ & $\begin{array}{l}14.322 \\
(0.281)\end{array}$ & $\begin{array}{l}0.951 \\
(0.622)\end{array}$ & $\begin{array}{l}0.651 \\
(0.764)\end{array}$ & 0.606 \\
\hline $94 / 1-96 / 12$ & $\begin{array}{l}0.005 \\
(0.312)\end{array}$ & $\begin{array}{l}0.615 \\
(0.000)^{* * *}\end{array}$ & $\begin{array}{l}0.353 \\
(0.000) * * *\end{array}$ & $\begin{array}{l}0.000 \\
(0.000)^{* * *}\end{array}$ & $\begin{array}{l}-0.175 \\
(0.039) * *\end{array}$ & $\begin{array}{l}1.008 \\
(0.000) * * *\end{array}$ & $\begin{array}{l}5.369 \\
(0.945)\end{array}$ & $\begin{array}{l}10.832 \\
(0.543)\end{array}$ & $\begin{array}{l}1.454 \\
(0.483)\end{array}$ & $\begin{array}{l}0.570 \\
(0.826)\end{array}$ & 0.530 \\
\hline $95 / 1-97 / 12$ & $\begin{array}{l}0.010 \\
(0.225)\end{array}$ & $\begin{array}{l}0.888 \\
(0.000) * * *\end{array}$ & $\begin{array}{l}0.056 \\
(0.808)\end{array}$ & $\begin{array}{l}0.000 \\
(0.000) * * *\end{array}$ & $\begin{array}{l}0.050 \\
(0.779)\end{array}$ & $\begin{array}{l}1.187 \\
(0.000) * * *\end{array}$ & $\begin{array}{l}10.460 \\
(0.576)\end{array}$ & $\begin{array}{l}9.527 \\
(0.657)\end{array}$ & $\begin{array}{l}1.040 \\
(0.594)\end{array}$ & $\begin{array}{l}0.564 \\
(0.831)\end{array}$ & 0.643 \\
\hline $96 / 1-98 / 12$ & $\begin{array}{l}0.012 \\
(0.257)\end{array}$ & $\begin{array}{l}0.842 \\
(0.000) * * *\end{array}$ & $\begin{array}{l}-0.185 \\
(0.475)\end{array}$ & $\begin{array}{l}0.000 \\
(0.946)\end{array}$ & $\begin{array}{l}0.253 \\
(0.258)\end{array}$ & $\begin{array}{l}0.854 \\
(0.000)^{* * *}\end{array}$ & $\begin{array}{l}8.034 \\
(0.782)\end{array}$ & $\begin{array}{l}7.032 \\
(0.856)\end{array}$ & $\begin{array}{l}1.006 \\
(0.605)\end{array}$ & $\begin{array}{l}0.325 \\
(0.967)\end{array}$ & 0.535 \\
\hline $97 / 1-99 / 12$ & $\begin{array}{l}0.000 \\
(0.969)\end{array}$ & $\begin{array}{l}1.041 \\
(0.000) * * *\end{array}$ & $\begin{array}{l}-0.713 \\
(0.009) * * *\end{array}$ & $\begin{array}{l}0.001 \\
(0.000) * * *\end{array}$ & $\begin{array}{l}-0.205 \\
(0.089)^{*}\end{array}$ & $\begin{array}{l}1.106 \\
(0.000) * * *\end{array}$ & $\begin{array}{l}8.320 \\
(0.760)\end{array}$ & $\begin{array}{l}13.270 \\
(0.350)\end{array}$ & $\begin{array}{l}1.413 \\
(0.493)\end{array}$ & $\begin{array}{l}0.717 \\
(0.712)\end{array}$ & 0.563 \\
\hline $98 / 1-00 / 12$ & $\begin{array}{l}0.003 \\
(0.768)\end{array}$ & $\begin{array}{l}0.794 \\
(0.000) * * *\end{array}$ & $\begin{array}{l}-0.637 \\
(0.011)^{* *}\end{array}$ & $\begin{array}{l}0.007 \\
(0.000) * * *\end{array}$ & $\begin{array}{l}-0.272 \\
(0.015)^{* *}\end{array}$ & $\begin{array}{l}0.684 \\
(0.000) * * *\end{array}$ & $\begin{array}{l}8.041 \\
(0.782)\end{array}$ & $\begin{array}{l}12.549 \\
(0.403)\end{array}$ & $\begin{array}{l}0.002 \\
(0.999)\end{array}$ & $\begin{array}{l}0.659 \\
(0.758)\end{array}$ & 0.297 \\
\hline $99 / 1-01 / 12$ & $\begin{array}{l}0.033 \\
(0.001) * * *\end{array}$ & $\begin{array}{l}0.400 \\
(0.000) * * *\end{array}$ & $\begin{array}{l}0.380 \\
(0.001)^{* * *}\end{array}$ & $\begin{array}{l}0.000 \\
(0.000) * * *\end{array}$ & $\begin{array}{l}-0.116 \\
(0.642)\end{array}$ & $\begin{array}{l}1.143 \\
(0.000) * * *\end{array}$ & $\begin{array}{l}10.719 \\
(0.553)\end{array}$ & $\begin{array}{l}15.445 \\
(0.218)\end{array}$ & $\begin{array}{l}1.147 \\
(0.564)\end{array}$ & $\begin{array}{l}1.465 \\
(0.267)\end{array}$ & 0.221 \\
\hline $00 / 1-02 / 12$ & $\begin{array}{l}0.023 \\
(0.001)^{* * *}\end{array}$ & $\begin{array}{l}0.325 \\
(0.000)^{* * *}\end{array}$ & $\begin{array}{l}0.411 \\
(0.000)^{* * *}\end{array}$ & $\begin{array}{l}0.000 \\
(0.166)\end{array}$ & $\begin{array}{l}-0.143 \\
(0.642)\end{array}$ & $\begin{array}{l}1.071 \\
(0.006) * * *\end{array}$ & $\begin{array}{l}7.696 \\
(0.808)\end{array}$ & $\begin{array}{l}14.116 \\
(0.293)\end{array}$ & $\begin{array}{l}1.846 \\
(0.397)\end{array}$ & $\begin{array}{l}1.583 \\
(0.227)\end{array}$ & 0.317 \\
\hline $01 / 1-03 / 12$ & $\begin{array}{l}0.019 \\
(0.013) * *\end{array}$ & $\begin{array}{l}0.418 \\
(0.000) * * *\end{array}$ & $\begin{array}{l}0.317 \\
(0.013) * *\end{array}$ & $\begin{array}{l}0.002 \\
(0.309)\end{array}$ & $\begin{array}{l}-0.156 \\
(0.059) *\end{array}$ & $\begin{array}{l}0.354 \\
(0.624)\end{array}$ & $\begin{array}{l}3.663 \\
(0.989)\end{array}$ & $\begin{array}{l}6.761 \\
(0.873)\end{array}$ & $\begin{array}{l}0.865 \\
(0.649)\end{array}$ & $\begin{array}{l}0.377 \\
(0.946)\end{array}$ & 0.552 \\
\hline $02 / 1-04 / 11$ & $\begin{array}{l}-0.002 \\
(0.747)\end{array}$ & $\begin{array}{l}0.310 \\
(0.008) * * *\end{array}$ & $\begin{array}{l}0.551 \\
(0.000) * * *\end{array}$ & $\begin{array}{l}0.001 \\
(0.368)\end{array}$ & $\begin{array}{l}0.232 \\
(0.181)\end{array}$ & $\begin{array}{l}0.166 \\
(0.813)\end{array}$ & $\begin{array}{l}8.658 \\
(0.732)\end{array}$ & $\begin{array}{l}7.758 \\
(0.804)\end{array}$ & $\begin{array}{l}1.412 \\
(0.494)\end{array}$ & $\begin{array}{l}0.492 \\
(0.878)\end{array}$ & 0.683 \\
\hline Full Sample & $\begin{array}{l}0.002 \\
(0.654)\end{array}$ & $\begin{array}{l}0.522 \\
(0.000) * * *\end{array}$ & $\begin{array}{l}0.398 \\
(0.000) * * *\end{array}$ & $\begin{array}{l}0.000 \\
(0.270)\end{array}$ & $\begin{array}{l}0.159 \\
(0.042)^{* *}\end{array}$ & $\begin{array}{l}0.797 \\
(0.000) * * *\end{array}$ & $\begin{array}{l}7.872 \\
(0.795)\end{array}$ & $\begin{array}{l}9.846 \\
(0.629)\end{array}$ & $\begin{array}{l}0.700 \\
(0.705)\end{array}$ & $\begin{array}{l}0.722 \\
(0.729)\end{array}$ & 0.565 \\
\hline
\end{tabular}


Table 5: Rolling Regression Estimates of the GW-CAPM Model for Philippines

\begin{tabular}{|c|c|c|c|c|c|c|c|c|c|c|c|}
\hline & Constant Mean & Group & World & Constant Variance & $\mathrm{ARCH}$ & GARCH & $\mathrm{Q}(12)$ & $\mathrm{Q}^{2}(12)$ & Normality & ARCH LM & $\operatorname{Adj} R^{2}$ \\
\hline $88 / 1-90 / 12$ & $\begin{array}{l}-0.010 \\
(0.198)\end{array}$ & $\begin{array}{l}0.541 \\
(0.000)^{* * *}\end{array}$ & $\begin{array}{l}0.274 \\
(0.010)^{* *}\end{array}$ & $\begin{array}{l}0.003 \\
(0.177)\end{array}$ & $\begin{array}{l}-0.141 \\
(0.018)^{* *}\end{array}$ & $\begin{array}{l}0.567 \\
(0.116)\end{array}$ & $\begin{array}{l}12.357 \\
(0.417)\end{array}$ & $\begin{array}{l}19.316 \\
(0.081)^{*}\end{array}$ & $\begin{array}{l}2.794 \\
(0.247)\end{array}$ & $\begin{array}{l}1.548 \\
(0.239)\end{array}$ & 0.229 \\
\hline $89 / 1-91 / 12$ & $\begin{array}{l}0.008 \\
(0.363)\end{array}$ & $\begin{array}{l}0.616 \\
(0.000)^{* * *}\end{array}$ & $\begin{array}{l}0.385 \\
(0.004) * * *\end{array}$ & $\begin{array}{l}0.003 \\
(0.015)^{* *}\end{array}$ & $\begin{array}{l}-0.137 \\
(0.056)^{*}\end{array}$ & $\begin{array}{l}0.618 \\
(0.002) * * *\end{array}$ & $\begin{array}{l}15.643 \\
(0.208)\end{array}$ & $\begin{array}{l}15.265 \\
(0.227)\end{array}$ & $\begin{array}{l}6.775 \\
(0.034)^{* *}\end{array}$ & $\begin{array}{l}0.882 \\
(0.585)\end{array}$ & 0.399 \\
\hline $90 / 1-92 / 12$ & $\begin{array}{l}0.009 \\
(0.320)\end{array}$ & $\begin{array}{l}0.738 \\
(0.000)^{* * *}\end{array}$ & $\begin{array}{l}0.424 \\
(0.030) * *\end{array}$ & $\begin{array}{l}0.003 \\
(0.327)\end{array}$ & $\begin{array}{l}-0.096 \\
(0.085)^{* *}\end{array}$ & $\begin{array}{l}0.543 \\
(0.438)\end{array}$ & $\begin{array}{l}7.235 \\
(0.842)\end{array}$ & $\begin{array}{l}3.996 \\
(0.984)\end{array}$ & $\begin{array}{l}5.634 \\
(0.060)^{*}\end{array}$ & $\begin{array}{l}1.360 \\
(0.309)\end{array}$ & 0.570 \\
\hline $91 / 1-93 / 12$ & $\begin{array}{l}0.014 \\
(0.011)^{* *}\end{array}$ & $\begin{array}{l}1.091 \\
(0.000)^{* * *}\end{array}$ & $\begin{array}{l}0.122 \\
(0.303)\end{array}$ & $\begin{array}{l}0.001 \\
(0.147)\end{array}$ & $\begin{array}{l}-0.397 \\
(0.014)^{* *}\end{array}$ & $\begin{array}{l}1.077 \\
(0.000) * * *\end{array}$ & $\begin{array}{l}9.193 \\
(0.686)\end{array}$ & $\begin{array}{l}13.672 \\
(0.322)\end{array}$ & $\begin{array}{l}2.164 \\
(0.339)\end{array}$ & $\begin{array}{l}0.935 \\
(0.548)\end{array}$ & 0.626 \\
\hline $92 / 1-94 / 12$ & $\begin{array}{l}0.012 \\
(0.222)\end{array}$ & $\begin{array}{l}1.078 \\
(0.000)^{* * *}\end{array}$ & $\begin{array}{l}0.001 \\
(0.997)\end{array}$ & $\begin{array}{l}0.001 \\
(0.797)\end{array}$ & $\begin{array}{l}-0.054 \\
(0.738)\end{array}$ & $\begin{array}{l}0.766 \\
(0.460)\end{array}$ & $\begin{array}{l}15.482 \\
(0.216)\end{array}$ & $\begin{array}{l}16.378 \\
(0.175)\end{array}$ & $\begin{array}{l}1.347 \\
(0.510)\end{array}$ & $\begin{array}{l}1.533 \\
(0.244)\end{array}$ & 0.698 \\
\hline $93 / 1-95 / 12$ & $\begin{array}{l}-0.004 \\
(0.367)\end{array}$ & $\begin{array}{l}1.358 \\
(0.000)^{* * *}\end{array}$ & $\begin{array}{l}-0.490 \\
(0.000)^{* * *}\end{array}$ & $\begin{array}{l}0.001 \\
(0.000) * * *\end{array}$ & $\begin{array}{l}-0.310 \\
(0.001)^{* * *}\end{array}$ & $\begin{array}{l}1.004 \\
(0.000) * * *\end{array}$ & $\begin{array}{l}14.182 \\
(0.289)\end{array}$ & $\begin{array}{l}17.805 \\
(0.122)\end{array}$ & $\begin{array}{l}0.450 \\
(0.799)\end{array}$ & $\begin{array}{l}1.361 \\
(0.308)\end{array}$ & 0.750 \\
\hline $94 / 1-96 / 12$ & $\begin{array}{l}0.007 \\
(0.213)\end{array}$ & $\begin{array}{l}1.115 \\
(0.000)^{* * *}\end{array}$ & $\begin{array}{l}-0.292 \\
(0.015) * *\end{array}$ & $\begin{array}{l}0.000 \\
(0.000) * * *\end{array}$ & $\begin{array}{l}-0.189 \\
(0.352)\end{array}$ & $\begin{array}{l}1.117 \\
(0.000) * * *\end{array}$ & $\begin{array}{l}18.227 \\
(0.109)\end{array}$ & $\begin{array}{l}12.471 \\
(0.409)\end{array}$ & $\begin{array}{l}0.101 \\
(0.951)\end{array}$ & $\begin{array}{l}1.342 \\
(0.317)\end{array}$ & 0.677 \\
\hline $95 / 1-97 / 12$ & $\begin{array}{l}-0.012 \\
(0.227)\end{array}$ & $\begin{array}{l}0.916 \\
(0.000)^{* * *}\end{array}$ & $\begin{array}{l}0.302 \\
(0.271)\end{array}$ & $\begin{array}{l}0.000 \\
(0.320)\end{array}$ & $\begin{array}{l}-0.032 \\
(0.931)\end{array}$ & $\begin{array}{l}1.139 \\
(0.000)^{* * *}\end{array}$ & $\begin{array}{l}10.994 \\
(0.529)\end{array}$ & $\begin{array}{l}5.671 \\
(0.932)\end{array}$ & $\begin{array}{l}0.066 \\
(0.967)\end{array}$ & $\begin{array}{l}0.239 \\
(0.990)\end{array}$ & 0.542 \\
\hline $96 / 1-98 / 12$ & $\begin{array}{l}0.009 \\
(0.332)\end{array}$ & $\begin{array}{l}0.796 \\
(0.000)^{* * *}\end{array}$ & $\begin{array}{l}0.210 \\
(0.372)\end{array}$ & $\begin{array}{l}0.000 \\
(0.551)\end{array}$ & $\begin{array}{l}-0.119 \\
(0.563)\end{array}$ & $\begin{array}{l}1.142 \\
(0.000) * * *\end{array}$ & $\begin{array}{l}11.632 \\
(0.476)\end{array}$ & $\begin{array}{l}6.463 \\
(0.891)\end{array}$ & $\begin{array}{l}0.296 \\
(0.862)\end{array}$ & $\begin{array}{l}0.283 \\
(0.980)\end{array}$ & 0.640 \\
\hline $97 / 1-99 / 12$ & $\begin{array}{l}-0.023 \\
(0.055)^{*}\end{array}$ & $\begin{array}{l}0.610 \\
(0.000)^{* * *}\end{array}$ & $\begin{array}{l}0.671 \\
(0.007) * * *\end{array}$ & $\begin{array}{l}0.006 \\
(0.334)\end{array}$ & $\begin{array}{l}-0.104 \\
(0.319)\end{array}$ & $\begin{array}{l}0.044 \\
(0.969)\end{array}$ & $\begin{array}{l}16.154 \\
(0.184)\end{array}$ & $\begin{array}{l}7.253 \\
(0.840)\end{array}$ & $\begin{array}{l}0.151 \\
(0.927)\end{array}$ & $\begin{array}{l}0.501 \\
(0.874)\end{array}$ & 0.667 \\
\hline $98 / 1-00 / 12$ & $\begin{array}{l}-0.028 \\
(0.000) * * *\end{array}$ & $\begin{array}{l}0.588 \\
(0.000)^{* * *}\end{array}$ & $\begin{array}{l}0.908 \\
(0.000) * * *\end{array}$ & $\begin{array}{l}0.000 \\
(0.000) * * *\end{array}$ & $\begin{array}{l}-0.194 \\
(0.090)^{* *}\end{array}$ & $\begin{array}{l}1.166 \\
(0.000) * * *\end{array}$ & $\begin{array}{l}8.545 \\
(0.741)\end{array}$ & $\begin{array}{l}9.897 \\
(0.625)\end{array}$ & $\begin{array}{l}1.139 \\
(0.566)\end{array}$ & $\begin{array}{l}2.177 \\
(0.104)\end{array}$ & 0.696 \\
\hline $99 / 1-01 / 12$ & $\begin{array}{l}-0.034 \\
(0.000) * * *\end{array}$ & $\begin{array}{l}0.663 \\
(0.000) * * *\end{array}$ & $\begin{array}{l}0.521 \\
(0.000) * * *\end{array}$ & $\begin{array}{l}0.002 \\
(0.017)^{* *}\end{array}$ & $\begin{array}{l}0.662 \\
(0.002)^{* * *}\end{array}$ & $\begin{array}{l}-0.300 \\
(0.125)\end{array}$ & $\begin{array}{l}6.669 \\
(0.879)\end{array}$ & $\begin{array}{l}14.126 \\
(0.293)\end{array}$ & $\begin{array}{l}2.244 \\
(0.326)\end{array}$ & $\begin{array}{l}0.541 \\
(0.847)\end{array}$ & 0.713 \\
\hline $00 / 1-02 / 12$ & $\begin{array}{l}-0.016 \\
(0.000) * * *\end{array}$ & $\begin{array}{l}0.790 \\
(0.000)^{* * *}\end{array}$ & $\begin{array}{l}0.317 \\
(0.000) * * *\end{array}$ & $\begin{array}{l}0.005 \\
(0.001) * * *\end{array}$ & $\begin{array}{l}0.659 \\
(0.000)^{* * *}\end{array}$ & $\begin{array}{l}-0.659 \\
(0.000) * * *\end{array}$ & $\begin{array}{l}10.109 \\
(0.606)\end{array}$ & $\begin{array}{l}10.568 \\
(0.566)\end{array}$ & $\begin{array}{l}0.951 \\
(0.622)\end{array}$ & $\begin{array}{l}0.638 \\
(0.774)\end{array}$ & 0.595 \\
\hline $01 / 1-03 / 12$ & $\begin{array}{l}-0.026 \\
(0.003)^{* * *}\end{array}$ & $\begin{array}{l}0.934 \\
(0.000)^{* * *}\end{array}$ & $\begin{array}{l}0.342 \\
(0.009) * * *\end{array}$ & $\begin{array}{l}0.000 \\
(0.000)^{* * *}\end{array}$ & $\begin{array}{l}-0.203 \\
(0.189)\end{array}$ & $\begin{array}{l}1.177 \\
(0.000) * * *\end{array}$ & $\begin{array}{l}12.926 \\
(0.374)\end{array}$ & $\begin{array}{l}5.538 \\
(0.938)\end{array}$ & $\begin{array}{l}1.830 \\
(0.401)\end{array}$ & $\begin{array}{l}0.348 \\
(0.959)\end{array}$ & 0.610 \\
\hline $02 / 1-04 / 11$ & $\begin{array}{l}-0.023 \\
(0.001)^{* * *}\end{array}$ & $\begin{array}{l}0.912 \\
(0.000)^{* * *}\end{array}$ & $\begin{array}{l}-0.034 \\
(0.851)\end{array}$ & $\begin{array}{l}0.001 \\
(0.000)^{* * *}\end{array}$ & $\begin{array}{l}-0.305 \\
(0.209)\end{array}$ & $\begin{array}{l}1.190 \\
(0.000) * * *\end{array}$ & $\begin{array}{l}12.214 \\
(0.429)\end{array}$ & $\begin{array}{l}10.476 \\
(0.574)\end{array}$ & $\begin{array}{l}1.857 \\
(0.395)\end{array}$ & $\begin{array}{l}0.826 \\
(0.628)\end{array}$ & 0.551 \\
\hline Full Sample & $\begin{array}{l}-0.003 \\
(0.493)\end{array}$ & $\begin{array}{l}0.732 \\
(0.000)^{* * *}\end{array}$ & $\begin{array}{l}0.341 \\
(0.000) * * *\end{array}$ & $\begin{array}{l}0.003 \\
(0.938) \\
\end{array}$ & $\begin{array}{l}-0.004 \\
(0.934)\end{array}$ & $\begin{array}{l}0.334 \\
(0.969) \\
\end{array}$ & $\begin{array}{l}10.383 \\
(0.582) \\
\end{array}$ & $\begin{array}{l}27.781 \\
(0.006) * * *\end{array}$ & $\begin{array}{l}1.656 \\
(0.437)\end{array}$ & $\begin{array}{l}2.380 \\
(0.007) * * *\end{array}$ & 0.637 \\
\hline
\end{tabular}


Table 6: Rolling Regression Estimates of the GW-CAPM Model for Singapore

\begin{tabular}{|c|c|c|c|c|c|c|c|c|c|c|c|}
\hline & Constant Mean & Group & World & Constant Variance & $\mathrm{ARCH}$ & GARCH & $\mathrm{Q}(12)$ & $\mathrm{Q}^{2}(12)$ & Normality & ARCH LM & $\operatorname{Adj} R^{2}$ \\
\hline $88 / 1-90 / 12$ & $\begin{array}{l}0.002 \\
(0.691)\end{array}$ & $\begin{array}{l}0.378 \\
(0.000)^{* * *}\end{array}$ & $\begin{array}{l}0.615 \\
(0.000) * * *\end{array}$ & $\begin{array}{l}0.001 \\
(0.004)^{* * * *}\end{array}$ & $\begin{array}{l}-0.207 \\
(0.284)\end{array}$ & $\begin{array}{l}0.770 \\
(0.002)^{* * *}\end{array}$ & $\begin{array}{l}16.504 \\
(0.169)\end{array}$ & $\begin{array}{l}11.278 \\
(0.505)\end{array}$ & $\begin{array}{l}1.042 \\
(0.594)\end{array}$ & $\begin{array}{l}0.594 \\
(0.808)\end{array}$ & 0.720 \\
\hline $89 / 1-91 / 12$ & $\begin{array}{l}0.006 \\
(0.286)\end{array}$ & $\begin{array}{l}0.400 \\
(0.000)^{* * *}\end{array}$ & $\begin{array}{l}0.631 \\
(0.000) * * *\end{array}$ & $\begin{array}{l}0.000 \\
(0.484)\end{array}$ & $\begin{array}{l}0.337 \\
(0.467)\end{array}$ & $\begin{array}{l}0.507 \\
(0.300)\end{array}$ & $\begin{array}{l}12.093 \\
(0.438)\end{array}$ & $\begin{array}{l}8.062 \\
(0.780)\end{array}$ & $\begin{array}{l}3.799 \\
(0.150)\end{array}$ & $\begin{array}{l}0.501 \\
(0.874)\end{array}$ & 0.816 \\
\hline $90 / 1-92 / 12$ & $\begin{array}{l}0.003 \\
(0.330)\end{array}$ & $\begin{array}{l}0.427 \\
(0.000)^{* * *}\end{array}$ & $\begin{array}{l}0.567 \\
(0.000) * * *\end{array}$ & $\begin{array}{l}0.000 \\
(0.000)^{* * *}\end{array}$ & $\begin{array}{l}-0.167 \\
(0.003) * * *\end{array}$ & $\begin{array}{l}1.070 \\
(0.000)^{* * *}\end{array}$ & $\begin{array}{l}9.140 \\
(0.691)\end{array}$ & $\begin{array}{l}5.987 \\
(0.917)\end{array}$ & $\begin{array}{l}0.636 \\
(0.727)\end{array}$ & $\begin{array}{l}0.585 \\
(0.814)\end{array}$ & 0.911 \\
\hline $91 / 1-93 / 12$ & $\begin{array}{l}0.004 \\
(0.245)\end{array}$ & $\begin{array}{l}0.427 \\
(0.000)^{* * *}\end{array}$ & $\begin{array}{l}0.570 \\
(0.000) * * *\end{array}$ & $\begin{array}{l}0.000 \\
(0.000)^{* * *}\end{array}$ & $\begin{array}{l}-0.114 \\
(0.696)\end{array}$ & $\begin{array}{l}1.207 \\
(0.000)^{* * *}\end{array}$ & $\begin{array}{l}9.054 \\
(0.698)\end{array}$ & $\begin{array}{l}5.107 \\
(0.954)\end{array}$ & $\begin{array}{l}1.691 \\
(0.429)\end{array}$ & $\begin{array}{l}0.730 \\
(0.702)\end{array}$ & 0.877 \\
\hline $92 / 1-94 / 12$ & $\begin{array}{l}0.002 \\
(0.571)\end{array}$ & $\begin{array}{l}0.399 \\
(0.000)^{* * *}\end{array}$ & $\begin{array}{l}0.569 \\
(0.000) * * *\end{array}$ & $\begin{array}{l}0.000 \\
(0.000)^{* * *}\end{array}$ & $\begin{array}{l}-0.164 \\
(0.399)\end{array}$ & $\begin{array}{l}1.160 \\
(0.000)^{* * *}\end{array}$ & $\begin{array}{l}4.780 \\
(0.965)\end{array}$ & $\begin{array}{l}13.290 \\
(0.348)\end{array}$ & $\begin{array}{l}2.028 \\
(0.363)\end{array}$ & $\begin{array}{l}1.308 \\
(0.332)\end{array}$ & 0.839 \\
\hline $93 / 1-95 / 12$ & $\begin{array}{l}0.004 \\
(0.262)\end{array}$ & $\begin{array}{l}0.512 \\
(0.000)^{* * *}\end{array}$ & $\begin{array}{l}0.470 \\
(0.000)^{* * *}\end{array}$ & $\begin{array}{l}0.000 \\
(0.000)^{* * *}\end{array}$ & $\begin{array}{l}-0.171 \\
(0.233)\end{array}$ & $\begin{array}{l}1.138 \\
(0.000)^{* * *}\end{array}$ & $\begin{array}{l}4.627 \\
(0.969)\end{array}$ & $\begin{array}{l}18.196 \\
(0.110)\end{array}$ & $\begin{array}{l}1.273 \\
(0.529)\end{array}$ & $\begin{array}{l}0.844 \\
(0.614)\end{array}$ & 0.824 \\
\hline $94 / 1-96 / 12$ & $\begin{array}{l}-0.001 \\
(0.576)\end{array}$ & $\begin{array}{l}0.501 \\
(0.000)^{* * *}\end{array}$ & $\begin{array}{l}0.402 \\
(0.000)^{* * *}\end{array}$ & $\begin{array}{l}0.000 \\
(0.000)^{* * *}\end{array}$ & $\begin{array}{l}-0.185 \\
(0.471)\end{array}$ & $\begin{array}{l}1.144 \\
(0.000)^{* * *}\end{array}$ & $\begin{array}{l}14.895 \\
(0.247)\end{array}$ & $\begin{array}{l}14.963 \\
(0.243)\end{array}$ & $\begin{array}{l}0.421 \\
(0.810)\end{array}$ & $\begin{array}{l}1.363 \\
(0.308)\end{array}$ & 0.778 \\
\hline $95 / 1-97 / 12$ & $\begin{array}{l}-0.012 \\
(0.049) * *\end{array}$ & $\begin{array}{l}0.261 \\
(0.000)^{* * *}\end{array}$ & $\begin{array}{l}0.825 \\
(0.000) * * *\end{array}$ & $\begin{array}{l}0.000 \\
(0.000)^{* * *}\end{array}$ & $\begin{array}{l}0.034 \\
(0.888)\end{array}$ & $\begin{array}{l}1.169 \\
(0.000)^{* * *}\end{array}$ & $\begin{array}{l}12.082 \\
(0.439)\end{array}$ & $\begin{array}{l}15.700 \\
(0.205)\end{array}$ & $\begin{array}{l}1.042 \\
(0.594)\end{array}$ & $\begin{array}{l}1.129 \\
(0.424)\end{array}$ & 0.696 \\
\hline $96 / 1-98 / 12$ & $\begin{array}{l}-0.011 \\
(0.103)\end{array}$ & $\begin{array}{l}0.309 \\
(0.038)^{* *}\end{array}$ & $\begin{array}{l}0.771 \\
(0.000)^{* * *}\end{array}$ & $\begin{array}{l}0.000 \\
(0.293)\end{array}$ & $\begin{array}{l}0.295 \\
(0.253)\end{array}$ & $\begin{array}{l}0.749 \\
(0.000)^{* * *}\end{array}$ & $\begin{array}{l}13.477 \\
(0.335)\end{array}$ & $\begin{array}{l}14.261 \\
(0.284)\end{array}$ & $\begin{array}{l}2.244 \\
(0.326)\end{array}$ & $\begin{array}{l}0.430 \\
(0.919)\end{array}$ & 0.657 \\
\hline $97 / 1-99 / 12$ & $\begin{array}{l}0.012 \\
(0.143)\end{array}$ & $\begin{array}{l}0.426 \\
(0.000)^{* * *}\end{array}$ & $\begin{array}{l}0.729 \\
(0.000)^{* * *}\end{array}$ & $\begin{array}{l}0.000 \\
(0.473)\end{array}$ & $\begin{array}{l}0.280 \\
(0.286)\end{array}$ & $\begin{array}{l}0.630 \\
(0.015)^{* *}\end{array}$ & $\begin{array}{l}9.280 \\
(0.679)\end{array}$ & $\begin{array}{l}12.295 \\
(0.422)\end{array}$ & $\begin{array}{l}0.786 \\
(0.675)\end{array}$ & $\begin{array}{l}0.522 \\
(0.860)\end{array}$ & 0.684 \\
\hline $98 / 1-00 / 12$ & $\begin{array}{l}0.018 \\
(0.002)^{* * *}\end{array}$ & $\begin{array}{l}0.504 \\
(0.000)^{* * *}\end{array}$ & $\begin{array}{l}0.808 \\
(0.000)^{* * *}\end{array}$ & $\begin{array}{l}0.001 \\
(0.063)^{*}\end{array}$ & $\begin{array}{l}0.561 \\
(0.010)^{* *}\end{array}$ & $\begin{array}{l}0.192 \\
(0.188)\end{array}$ & $\begin{array}{l}6.761 \\
(0.873)\end{array}$ & $\begin{array}{l}13.868 \\
(0.309)\end{array}$ & $\begin{array}{l}1.424 \\
(0.491)\end{array}$ & $\begin{array}{l}0.938 \\
(0.545)\end{array}$ & 0.637 \\
\hline $99 / 1-01 / 12$ & $\begin{array}{l}0.006 \\
(0.467)\end{array}$ & $\begin{array}{l}0.372 \\
(0.000)^{* * *}\end{array}$ & $\begin{array}{l}0.409 \\
(0.004)^{* * *}\end{array}$ & $\begin{array}{l}0.004 \\
(0.078)^{*}\end{array}$ & $\begin{array}{l}0.280 \\
(0.261)\end{array}$ & $\begin{array}{l}-0.452 \\
(0.254)\end{array}$ & $\begin{array}{l}19.134 \\
(0.085)^{*}\end{array}$ & $\begin{array}{l}9.508 \\
(0.659)\end{array}$ & $\begin{array}{l}1.998 \\
(0.368)\end{array}$ & $\begin{array}{l}0.338 \\
(0.962)\end{array}$ & 0.578 \\
\hline $00 / 1-02 / 12$ & $\begin{array}{l}0.002 \\
(0.693)\end{array}$ & $\begin{array}{l}0.239 \\
(0.000)^{* * *}\end{array}$ & $\begin{array}{l}0.432 \\
(0.000)^{* * *}\end{array}$ & $\begin{array}{l}0.001 \\
(0.001)^{* * *}\end{array}$ & $\begin{array}{l}1.144 \\
(0.001)^{* * *}\end{array}$ & $\begin{array}{l}-0.139 \\
(0.005)^{* * *}\end{array}$ & $\begin{array}{l}17.495 \\
(0.132)\end{array}$ & $\begin{array}{l}6.482 \\
(0.890)\end{array}$ & $\begin{array}{l}0.627 \\
(0.731)\end{array}$ & $\begin{array}{l}0.603 \\
(0.801)\end{array}$ & 0.537 \\
\hline $01 / 1-03 / 12$ & $\begin{array}{l}-0.003 \\
(0.459)\end{array}$ & $\begin{array}{l}0.309 \\
(0.000)^{* * *}\end{array}$ & $\begin{array}{l}0.446 \\
(0.000)^{* * *}\end{array}$ & $\begin{array}{l}0.002 \\
(0.004)^{* * *}\end{array}$ & $\begin{array}{l}0.547 \\
(0.024)^{* *}\end{array}$ & $\begin{array}{l}-0.384 \\
(0.021)^{* *}\end{array}$ & $\begin{array}{l}9.632 \\
(0.648)\end{array}$ & $\begin{array}{l}12.749 \\
(0.388)\end{array}$ & $\begin{array}{l}0.610 \\
(0.737)\end{array}$ & $\begin{array}{l}0.520 \\
(0.862)\end{array}$ & 0.644 \\
\hline $02 / 1-04 / 11$ & $\begin{array}{l}-0.001 \\
(0.709)\end{array}$ & $\begin{array}{l}0.402 \\
(0.000)^{* * *}\end{array}$ & $\begin{array}{l}0.567 \\
(0.000)^{* * *}\end{array}$ & $\begin{array}{l}0.000 \\
(0.000)^{* * *}\end{array}$ & $\begin{array}{l}-0.145 \\
(0.493)\end{array}$ & $\begin{array}{l}1.089 \\
(0.000)^{* * *}\end{array}$ & $\begin{array}{l}9.320 \\
(0.675)\end{array}$ & $\begin{array}{l}10.980 \\
(0.531)\end{array}$ & $\begin{array}{l}0.439 \\
(0.803)\end{array}$ & $\begin{array}{l}0.777 \\
(0.665)\end{array}$ & 0.811 \\
\hline Full Sample & $\begin{array}{l}0.002 \\
(0.520) \\
\end{array}$ & $\begin{array}{l}0.401 \\
(0.000)^{* * * *}\end{array}$ & $\begin{array}{l}0.572 \\
(0.000) * * *\end{array}$ & $\begin{array}{l}0.000 \\
(0.217)\end{array}$ & $\begin{array}{l}0.174 \\
(0.034) * *\end{array}$ & $\begin{array}{l}0.799 \\
(0.000) * * *\end{array}$ & $\begin{array}{l}11.058 \\
(0.524) \\
\end{array}$ & $\begin{array}{l}10.061 \\
(0.611) \\
\end{array}$ & $\begin{array}{l}3.139 \\
(0.208)\end{array}$ & $\begin{array}{l}0.864 \\
(0.585)\end{array}$ & 0.759 \\
\hline
\end{tabular}


Table 7: Rolling Regression Estimates of the GW-CAPM Model for Thailand

\begin{tabular}{|c|c|c|c|c|c|c|c|c|c|c|c|}
\hline & Constant Mean & Group & World & Constant Variance & $\mathrm{ARCH}$ & GARCH & $\mathrm{Q}(12)$ & $\mathrm{Q}^{2}(12)$ & Normality & ARCH LM & $\operatorname{Adj} R^{2}$ \\
\hline $88 / 1-90 / 12$ & $\begin{array}{l}0.005 \\
(0.504)\end{array}$ & $\begin{array}{l}0.676 \\
(0.000) * * *\end{array}$ & $\begin{array}{l}0.844 \\
(0.000)^{* * *}\end{array}$ & $\begin{array}{l}0.002 \\
(0.000) * * *\end{array}$ & $\begin{array}{l}-0.262 \\
(0.054)^{*}\end{array}$ & $\begin{array}{l}0.914 \\
(0.000)^{* * *}\end{array}$ & $\begin{array}{l}9.417 \\
(0.667)\end{array}$ & $\begin{array}{l}8.857 \\
(0.715)\end{array}$ & $\begin{array}{l}1.549 \\
(0.461)\end{array}$ & $\begin{array}{l}0.484 \\
(0.886)\end{array}$ & 0.498 \\
\hline $89 / 1-91 / 12$ & $\begin{array}{l}0.008 \\
(0.450)\end{array}$ & $\begin{array}{l}0.800 \\
(0.000)^{* * *}\end{array}$ & $\begin{array}{l}0.544 \\
(0.000)^{* * *}\end{array}$ & $\begin{array}{l}0.002 \\
(0.000) * * *\end{array}$ & $\begin{array}{l}-0.379 \\
(0.090)^{*}\end{array}$ & $\begin{array}{l}0.829 \\
(0.000)^{* * *}\end{array}$ & $\begin{array}{l}14.638 \\
(0.262)\end{array}$ & $\begin{array}{l}17.344 \\
(0.137)\end{array}$ & $\begin{array}{l}2.027 \\
(0.363)\end{array}$ & $\begin{array}{l}0.564 \\
(0.831)\end{array}$ & 0.620 \\
\hline $90 / 1-92 / 12$ & $\begin{array}{l}0.003 \\
(0.668)\end{array}$ & $\begin{array}{l}0.884 \\
(0.000) * * *\end{array}$ & $\begin{array}{l}0.316 \\
(0.094)^{*}\end{array}$ & $\begin{array}{l}0.001 \\
(0.000) * * *\end{array}$ & $\begin{array}{l}-0.298 \\
(0.001)^{* * *}\end{array}$ & $\begin{array}{l}1.079 \\
(0.000)^{* * *}\end{array}$ & $\begin{array}{l}16.278 \\
(0.179)\end{array}$ & $\begin{array}{l}10.486 \\
(0.573)\end{array}$ & $\begin{array}{l}1.761 \\
(0.414)\end{array}$ & $\begin{array}{l}0.992 \\
(0.509)\end{array}$ & 0.617 \\
\hline $91 / 1-93 / 12$ & $\begin{array}{l}0.014 \\
(0.061)^{*}\end{array}$ & $\begin{array}{l}1.068 \\
(0.000) * * *\end{array}$ & $\begin{array}{l}-0.118 \\
(0.300)\end{array}$ & $\begin{array}{l}0.002 \\
(0.000) * * *\end{array}$ & $\begin{array}{l}-0.348 \\
(0.013)^{* *}\end{array}$ & $\begin{array}{l}0.991 \\
(0.000)^{* * *}\end{array}$ & $\begin{array}{l}14.697 \\
(0.258)\end{array}$ & $\begin{array}{l}6.019 \\
(0.915)\end{array}$ & $\begin{array}{l}1.151 \\
(0.562)\end{array}$ & $\begin{array}{l}0.579 \\
(0.819)\end{array}$ & 0.504 \\
\hline $92 / 1-94 / 12$ & $\begin{array}{l}0.000 \\
(0.949)\end{array}$ & $\begin{array}{l}1.075 \\
(0.000) * * *\end{array}$ & $\begin{array}{l}-0.215 \\
(0.023) * *\end{array}$ & $\begin{array}{l}0.003 \\
(0.001) * * *\end{array}$ & $\begin{array}{l}-0.265 \\
(0.001)^{* * *}\end{array}$ & $\begin{array}{l}0.564 \\
(0.049)^{* *}\end{array}$ & $\begin{array}{l}22.854 \\
(0.029)^{* *}\end{array}$ & $\begin{array}{l}17.516 \\
(0.131)\end{array}$ & $\begin{array}{l}0.550 \\
(0.759)\end{array}$ & $\begin{array}{l}0.805 \\
(0.643)\end{array}$ & 0.638 \\
\hline $93 / 1-95 / 12$ & $\begin{array}{l}-0.004 \\
(0.291)\end{array}$ & $\begin{array}{l}1.198 \\
(0.000) * * *\end{array}$ & $\begin{array}{l}-0.161 \\
(0.057)^{*}\end{array}$ & $\begin{array}{l}0.000 \\
(0.000) * * *\end{array}$ & $\begin{array}{l}-0.162 \\
(0.409)\end{array}$ & $\begin{array}{l}1.096 \\
(0.000)^{* * *}\end{array}$ & $\begin{array}{l}13.749 \\
(0.317)\end{array}$ & $\begin{array}{l}19.978 \\
(0.068)^{*}\end{array}$ & $\begin{array}{l}1.111 \\
(0.574)\end{array}$ & $\begin{array}{l}0.637 \\
(0.775)\end{array}$ & 0.755 \\
\hline $94 / 1-96 / 12$ & $\begin{array}{l}-0.007 \\
(0.226)\end{array}$ & $\begin{array}{l}1.206 \\
(0.000)^{* * *}\end{array}$ & $\begin{array}{l}-0.116 \\
(0.386)\end{array}$ & $\begin{array}{l}0.000 \\
(0.670)\end{array}$ & $\begin{array}{l}-0.036 \\
(0.763)\end{array}$ & $\begin{array}{l}1.144 \\
(0.000)^{* * *}\end{array}$ & $\begin{array}{l}10.173 \\
(0.601)\end{array}$ & $\begin{array}{l}5.117 \\
(0.954)\end{array}$ & $\begin{array}{l}2.798 \\
(0.247)\end{array}$ & $\begin{array}{l}0.443 \\
(0.911)\end{array}$ & 0.676 \\
\hline $95 / 1-97 / 12$ & $\begin{array}{l}-0.010 \\
(0.017)^{* *}\end{array}$ & $\begin{array}{l}1.191 \\
(0.000) * * *\end{array}$ & $\begin{array}{l}-0.182 \\
(0.320)\end{array}$ & $\begin{array}{l}0.000 \\
(0.000) * * *\end{array}$ & $\begin{array}{l}-0.140 \\
(0.489)\end{array}$ & $\begin{array}{l}1.307 \\
(0.000)^{* * *}\end{array}$ & $\begin{array}{l}9.568 \\
(0.654)\end{array}$ & $\begin{array}{l}6.138 \\
(0.909)\end{array}$ & $\begin{array}{l}1.007 \\
(0.604)\end{array}$ & $\begin{array}{l}0.245 \\
(0.989)\end{array}$ & 0.554 \\
\hline $96 / 1-98 / 12$ & $\begin{array}{l}-0.049 \\
(0.000)^{* * *}\end{array}$ & $\begin{array}{l}0.641 \\
(0.000) * * *\end{array}$ & $\begin{array}{l}1.532 \\
(0.005)^{* * *}\end{array}$ & $\begin{array}{l}0.009 \\
(0.317)\end{array}$ & $\begin{array}{l}-0.107 \\
(0.138)\end{array}$ & $\begin{array}{l}0.498 \\
(0.474)\end{array}$ & $\begin{array}{l}10.731 \\
(0.552)\end{array}$ & $\begin{array}{l}15.678 \\
(0.206)\end{array}$ & $\begin{array}{l}6.493 \\
(0.039) * *\end{array}$ & $\begin{array}{l}0.665 \\
(0.753)\end{array}$ & 0.563 \\
\hline $97 / 1-99 / 12$ & $\begin{array}{l}-0.042 \\
(0.003) * * *\end{array}$ & $\begin{array}{l}0.680 \\
(0.000) * * *\end{array}$ & $\begin{array}{l}1.348 \\
(0.009)^{* * *}\end{array}$ & $\begin{array}{l}0.008 \\
(0.475)\end{array}$ & $\begin{array}{l}-0.100 \\
(0.157)\end{array}$ & $\begin{array}{l}0.506 \\
(0.582)\end{array}$ & $\begin{array}{l}10.550 \\
(0.568)\end{array}$ & $\begin{array}{l}14.459 \\
(0.272)\end{array}$ & $\begin{array}{l}2.705 \\
(0.259)\end{array}$ & $\begin{array}{l}0.381 \\
(0.944)\end{array}$ & 0.583 \\
\hline $98 / 1-00 / 12$ & $\begin{array}{l}-0.017 \\
(0.208)\end{array}$ & $\begin{array}{l}1.009 \\
(0.000) * * *\end{array}$ & $\begin{array}{l}0.671 \\
(0.005) * * *\end{array}$ & $\begin{array}{l}0.001 \\
(0.000)^{* * *}\end{array}$ & $\begin{array}{l}-0.174 \\
(0.000) * * *\end{array}$ & $\begin{array}{l}0.945 \\
(0.000) * * *\end{array}$ & $\begin{array}{l}20.034 \\
(0.066)^{*}\end{array}$ & $\begin{array}{l}8.504 \\
(0.745)\end{array}$ & $\begin{array}{l}1.490 \\
(0.475)\end{array}$ & $\begin{array}{l}0.674 \\
(0.746)\end{array}$ & 0.539 \\
\hline $99 / 1-01 / 12$ & $\begin{array}{l}-0.020 \\
(0.163)\end{array}$ & $\begin{array}{l}1.231 \\
(0.000)^{* * *}\end{array}$ & $\begin{array}{l}0.266 \\
(0.173)\end{array}$ & $\begin{array}{l}0.006 \\
(0.238)\end{array}$ & $\begin{array}{l}0.188 \\
(0.405)\end{array}$ & $\begin{array}{l}-0.185 \\
(0.829)\end{array}$ & $\begin{array}{l}60.674 \\
(0.000)^{* * *}\end{array}$ & $\begin{array}{l}6.622 \\
(0.882)\end{array}$ & $\begin{array}{l}0.938 \\
(0.626)\end{array}$ & $\begin{array}{l}0.760 \\
(0.679)\end{array}$ & 0.704 \\
\hline $00 / 1-02 / 12$ & $\begin{array}{l}-0.002 \\
(0.832)\end{array}$ & $\begin{array}{l}1.162 \\
(0.000) * * *\end{array}$ & $\begin{array}{l}0.172 \\
(0.208)\end{array}$ & $\begin{array}{l}0.000 \\
(0.107)\end{array}$ & $\begin{array}{l}-0.175 \\
(0.278)\end{array}$ & $\begin{array}{l}1.096 \\
(0.000)^{* * *}\end{array}$ & $\begin{array}{l}15.731 \\
(0.204)\end{array}$ & $\begin{array}{l}12.514 \\
(0.405)\end{array}$ & $\begin{array}{l}0.919 \\
(0.632)\end{array}$ & $\begin{array}{l}0.665 \\
(0.753)\end{array}$ & 0.630 \\
\hline $01 / 1-03 / 12$ & $\begin{array}{l}0.022 \\
(0.016)^{* *}\end{array}$ & $\begin{array}{l}0.677 \\
(0.000)^{* * *}\end{array}$ & $\begin{array}{l}0.407 \\
(0.008)^{* * *}\end{array}$ & $\begin{array}{l}0.000 \\
(0.305)\end{array}$ & $\begin{array}{l}-0.152 \\
(0.476)\end{array}$ & $\begin{array}{l}1.086 \\
(0.000)^{* * *}\end{array}$ & $\begin{array}{l}7.768 \\
(0.803)\end{array}$ & $\begin{array}{l}6.339 \\
(0.898)\end{array}$ & $\begin{array}{l}1.771 \\
(0.413)\end{array}$ & $\begin{array}{l}0.418 \\
(0.925)\end{array}$ & 0.511 \\
\hline $02 / 1-04 / 11$ & $\begin{array}{l}0.020 \\
(0.044)^{* *}\end{array}$ & $\begin{array}{l}0.519 \\
(0.004)^{* * *}\end{array}$ & $\begin{array}{l}0.621 \\
(0.001)^{* * *}\end{array}$ & $\begin{array}{l}0.002 \\
(0.632)\end{array}$ & $\begin{array}{l}0.095 \\
(0.731)\end{array}$ & $\begin{array}{l}0.348 \\
(0.763)\end{array}$ & $\begin{array}{l}8.272 \\
(0.764)\end{array}$ & $\begin{array}{l}5.803 \\
(0.926)\end{array}$ & $\begin{array}{l}10.098 \\
(0.006)^{* * *}\end{array}$ & $\begin{array}{l}0.282 \\
(0.979)\end{array}$ & 0.653 \\
\hline Full Sample & $\begin{array}{l}0.000 \\
(0.972)\end{array}$ & $\begin{array}{l}0.880 \\
(0.000) * * *\end{array}$ & $\begin{array}{l}0.335 \\
(0.007)^{* * *}\end{array}$ & $\begin{array}{l}0.000 \\
(0.355)\end{array}$ & $\begin{array}{l}0.103 \\
(0.115)\end{array}$ & $\begin{array}{l}0.848 \\
(0.000) * * *\end{array}$ & $\begin{array}{l}10.265 \\
(0.593)\end{array}$ & $\begin{array}{l}12.251 \\
(0.426)\end{array}$ & $\begin{array}{l}29.167 \\
(0.000)^{* * *}\end{array}$ & $\begin{array}{l}1.001 \\
(0.450)\end{array}$ & 0.617 \\
\hline
\end{tabular}




\begin{tabular}{|c|c|c|c|c|c|c|c|c|c|c|c|}
\hline & Constant Mean & Group & Region & Constant Variance & $\mathrm{ARCH}$ & GARCH & $\mathrm{Q}(12)$ & $\mathrm{Q}^{2}(12)$ & Normality & $\mathrm{ARCH}$ LM & $\operatorname{Adi} R^{2}$ \\
\hline $88 / 1-90 / 12$ & $\begin{array}{l}0.000 \\
(0.933)\end{array}$ & $\begin{array}{l}0.866 \\
(0.000) * * *\end{array}$ & $\begin{array}{l}0.353 \\
(0.003) * * *\end{array}$ & $\begin{array}{l}0.005 \\
(0.005)^{* * *}\end{array}$ & $\begin{array}{l}0.291 \\
(0.056) *\end{array}$ & $\begin{array}{l}-0.458 \\
(0.042)^{* *}\end{array}$ & $\begin{array}{l}20.305 \\
(0.062)^{*}\end{array}$ & $\begin{array}{l}9.956 \\
(0.620)\end{array}$ & $\begin{array}{l}1.463 \\
(0.481)\end{array}$ & $\begin{array}{l}1.637 \\
(0.212)\end{array}$ & 0.430 \\
\hline $89 / 1-91 / 12$ & $\begin{array}{l}0.018 \\
(0.023)^{* *}\end{array}$ & $\begin{array}{l}0.858 \\
(0.000) * * *\end{array}$ & $\begin{array}{l}0.359 \\
(0.000)^{* * *}\end{array}$ & $\begin{array}{l}0.001 \\
(0.000)^{* * *}\end{array}$ & $\begin{array}{l}-0.341 \\
(0.054)^{*}\end{array}$ & $\begin{array}{l}1.054 \\
(0.000) * * *\end{array}$ & $\begin{array}{l}10.837 \\
(0.543)\end{array}$ & $\begin{array}{l}11.527 \\
(0.484)\end{array}$ & $\begin{array}{l}2.675 \\
(0.262)\end{array}$ & $\begin{array}{l}0.572 \\
(0.824)\end{array}$ & 0.601 \\
\hline $90 / 1-92 / 12$ & $\begin{array}{l}0.011 \\
(0.145)\end{array}$ & $\begin{array}{l}0.943 \\
(0.000) * * *\end{array}$ & $\begin{array}{l}0.159 \\
(0.090) *\end{array}$ & $\begin{array}{l}0.001 \\
(0.000) * * *\end{array}$ & $\begin{array}{l}-0.292 \\
(0.052)^{*}\end{array}$ & $\begin{array}{l}1.127 \\
(0.000) * * *\end{array}$ & $\begin{array}{l}21.705 \\
(0.041)^{* *}\end{array}$ & $\begin{array}{l}11.456 \\
(0.490)\end{array}$ & $\begin{array}{l}2.349 \\
(0.309)\end{array}$ & $\begin{array}{l}0.804 \\
(0.644)\end{array}$ & 0.619 \\
\hline $91 / 1-93 / 12$ & $\begin{array}{l}0.010 \\
(0.144)\end{array}$ & $\begin{array}{l}0.999 \\
(0.000)^{* * *}\end{array}$ & $\begin{array}{l}-0.074 \\
(0.204)\end{array}$ & $\begin{array}{l}0.002 \\
(0.000) * * *\end{array}$ & $\begin{array}{l}-0.320 \\
(0.000)^{* * *}\end{array}$ & $\begin{array}{l}0.808 \\
(0.000)^{* * *}\end{array}$ & $\begin{array}{l}18.156 \\
(0.111)\end{array}$ & $\begin{array}{l}7.207 \\
(0.844)\end{array}$ & $\begin{array}{l}1.436 \\
(0.488)\end{array}$ & $\begin{array}{l}0.371 \\
(0.949)\end{array}$ & 0.513 \\
\hline $92 / 1-94 / 12$ & $\begin{array}{l}0.008 \\
(0.304)\end{array}$ & $\begin{array}{l}1.234 \\
(0.000)^{* * *}\end{array}$ & $\begin{array}{l}-0.133 \\
(0.214)\end{array}$ & $\begin{array}{l}0.000 \\
(0.000) * * *\end{array}$ & $\begin{array}{l}-0.020 \\
(0.916)\end{array}$ & $\begin{array}{l}1.050 \\
(0.000)^{* * *}\end{array}$ & $\begin{array}{l}12.102 \\
(0.438)\end{array}$ & $\begin{array}{l}13.001 \\
(0.369)\end{array}$ & $\begin{array}{l}0.494 \\
(0.781)\end{array}$ & $\begin{array}{l}0.673 \\
(0.747)\end{array}$ & 0.642 \\
\hline $93 / 1-95 / 12$ & $\begin{array}{l}-0.006 \\
(0.141)\end{array}$ & $\begin{array}{l}1.224 \\
(0.000)^{* * * *}\end{array}$ & $\begin{array}{l}-0.182 \\
(0.002) * * *\end{array}$ & $\begin{array}{l}0.000 \\
(0.000)^{* * *}\end{array}$ & $\begin{array}{l}-0.162 \\
(0.448)\end{array}$ & $\begin{array}{l}1.099 \\
(0.000)^{* * *}\end{array}$ & $\begin{array}{l}17.121 \\
(0.145)\end{array}$ & $\begin{array}{l}26.717 \\
(0.008) * * *\end{array}$ & $\begin{array}{l}1.592 \\
(0.451)\end{array}$ & $\begin{array}{l}0.840 \\
(0.617)\end{array}$ & 0.769 \\
\hline $94 / 1-96 / 12$ & $\begin{array}{l}-0.011 \\
(0.022) * *\end{array}$ & $\begin{array}{l}1.148 \\
(0.000)^{* * * *}\end{array}$ & $\begin{array}{l}-0.143 \\
(0.108)\end{array}$ & $\begin{array}{l}0.000 \\
(0.002) * * *\end{array}$ & $\begin{array}{l}-0.081 \\
(0.316)\end{array}$ & $\begin{array}{l}1.178 \\
(0.000)^{* * *}\end{array}$ & $\begin{array}{l}7.821 \\
(0.799)\end{array}$ & $\begin{array}{l}6.170 \\
(0.907)\end{array}$ & $\begin{array}{l}2.210 \\
(0.331)\end{array}$ & $\begin{array}{l}0.529 \\
(0.855)\end{array}$ & 0.671 \\
\hline $95 / 1-97 / 12$ & $\begin{array}{l}-0.013 \\
(0.001)^{* * *}\end{array}$ & $\begin{array}{l}1.154 \\
(0.000)^{* * *}\end{array}$ & $\begin{array}{l}-0.194 \\
(0.045)^{* *}\end{array}$ & $\begin{array}{l}0.000 \\
(0.714)\end{array}$ & $\begin{array}{l}-0.088 \\
(0.525)\end{array}$ & $\begin{array}{l}1.228 \\
(0.000)^{* * *}\end{array}$ & $\begin{array}{l}8.336 \\
(0.758)\end{array}$ & $\begin{array}{l}5.517 \\
(0.938)\end{array}$ & $\begin{array}{l}0.403 \\
(0.818)\end{array}$ & $\begin{array}{l}0.272 \\
(0.983)\end{array}$ & 0.553 \\
\hline $96 / 1-98 / 12$ & $\begin{array}{l}-0.032 \\
(0.005)^{* * *}\end{array}$ & $\begin{array}{l}0.725 \\
(0.000)^{* * *}\end{array}$ & $\begin{array}{l}0.948 \\
(0.000) * * *\end{array}$ & $\begin{array}{l}0.001 \\
(0.000)^{* * *}\end{array}$ & $\begin{array}{l}-0.145 \\
(0.270)\end{array}$ & $\begin{array}{l}1.051 \\
(0.000)^{* * *}\end{array}$ & $\begin{array}{l}14.262 \\
(0.284)\end{array}$ & $\begin{array}{l}16.407 \\
(0.173)\end{array}$ & $\begin{array}{l}7.825 \\
(0.020)^{* *}\end{array}$ & $\begin{array}{l}3.039 \\
(0.038) * *\end{array}$ & 0.570 \\
\hline $97 / 1-99 / 12$ & $\begin{array}{l}-0.014 \\
(0.416)\end{array}$ & $\begin{array}{l}0.675 \\
(0.000) * * *\end{array}$ & $\begin{array}{l}1.011 \\
(0.002) * * *\end{array}$ & $\begin{array}{l}0.008 \\
(0.031)^{* *}\end{array}$ & $\begin{array}{l}-0.129 \\
(0.134)\end{array}$ & $\begin{array}{l}0.552 \\
(0.129)\end{array}$ & $\begin{array}{l}12.756 \\
(0.387)\end{array}$ & $\begin{array}{l}17.292 \\
(0.139)\end{array}$ & $\begin{array}{l}3.743 \\
(0.154)\end{array}$ & $\begin{array}{l}0.879 \\
(0.588)\end{array}$ & 0.590 \\
\hline $98 / 1-00 / 12$ & $\begin{array}{l}-0.022 \\
(0.089)^{*}\end{array}$ & $\begin{array}{l}0.717 \\
(0.000)^{* * *}\end{array}$ & $\begin{array}{l}0.920 \\
(0.000) * * *\end{array}$ & $\begin{array}{l}0.005 \\
(0.000) * * *\end{array}$ & $\begin{array}{l}-0.145 \\
(0.001)^{* * *}\end{array}$ & $\begin{array}{l}0.607 \\
(0.000) * * *\end{array}$ & $\begin{array}{l}11.455 \\
(0.490)\end{array}$ & $\begin{array}{l}4.333 \\
(0.977)\end{array}$ & $\begin{array}{l}0.949 \\
(0.622)\end{array}$ & $\begin{array}{l}0.766 \\
(0.674)\end{array}$ & 0.602 \\
\hline $99 / 1-01 / 12$ & $\begin{array}{l}-0.021 \\
(0.134)\end{array}$ & $\begin{array}{l}1.169 \\
(0.000)^{* * *}\end{array}$ & $\begin{array}{l}0.405 \\
(0.039)^{* *}\end{array}$ & $\begin{array}{l}0.004 \\
(0.231)\end{array}$ & $\begin{array}{l}0.206 \\
(0.343)\end{array}$ & $\begin{array}{l}0.054 \\
(0.939)\end{array}$ & $\begin{array}{l}50.657 \\
(0.000) * * *\end{array}$ & $\begin{array}{l}8.980 \\
(0.705)\end{array}$ & $\begin{array}{l}1.140 \\
(0.565)\end{array}$ & $\begin{array}{l}0.791 \\
(0.654)\end{array}$ & 0.724 \\
\hline $00 / 1-02 / 12$ & $\begin{array}{l}-0.015 \\
(0.082)^{*}\end{array}$ & $\begin{array}{l}1.274 \\
(0.000)^{* * *}\end{array}$ & $\begin{array}{l}0.273 \\
(0.033)^{* *}\end{array}$ & $\begin{array}{l}0.010 \\
(0.000) * * *\end{array}$ & $\begin{array}{l}0.419 \\
(0.000) * * *\end{array}$ & $\begin{array}{l}-0.823 \\
(0.000)^{* * *}\end{array}$ & $\begin{array}{l}14.436 \\
(0.274)\end{array}$ & $\begin{array}{l}10.586 \\
(0.565)\end{array}$ & $\begin{array}{l}0.896 \\
(0.639)\end{array}$ & $\begin{array}{l}0.483 \\
(0.887)\end{array}$ & 0.640 \\
\hline $01 / 1-03 / 12$ & $\begin{array}{l}0.018 \\
(0.049) * *\end{array}$ & $\begin{array}{l}0.575 \\
(0.000)^{* * *}\end{array}$ & $\begin{array}{l}0.524 \\
(0.000) * * *\end{array}$ & $\begin{array}{l}0.000 \\
(0.416)\end{array}$ & $\begin{array}{l}-0.197 \\
(0.559)\end{array}$ & $\begin{array}{l}1.125 \\
(0.003) * * *\end{array}$ & $\begin{array}{l}8.849 \\
(0.716)\end{array}$ & $\begin{array}{l}2.688 \\
(0.997)\end{array}$ & $\begin{array}{l}3.089 \\
(0.213)\end{array}$ & $\begin{array}{l}0.521 \\
(0.861)\end{array}$ & 0.525 \\
\hline $02 / 1-04 / 11$ & $\begin{array}{l}0.018 \\
(0.054)^{*}\end{array}$ & $\begin{array}{l}0.701 \\
(0.000)^{* * *}\end{array}$ & $\begin{array}{l}0.439 \\
(0.028) * *\end{array}$ & $\begin{array}{l}0.002 \\
(0.529)\end{array}$ & $\begin{array}{l}0.230 \\
(0.285)\end{array}$ & $\begin{array}{l}0.251 \\
(0.741)\end{array}$ & $\begin{array}{l}8.806 \\
(0.719)\end{array}$ & $\begin{array}{l}4.628 \\
(0.969)\end{array}$ & $\begin{array}{l}10.061 \\
(0.007) * * *\end{array}$ & $\begin{array}{l}0.258 \\
(0.985)\end{array}$ & 0.622 \\
\hline Total & $\begin{array}{l}0.001 \\
(0.857)\end{array}$ & $\begin{array}{l}0.933 \\
(0.000) * * *\end{array}$ & $\begin{array}{l}0.247 \\
(0.010)^{* *}\end{array}$ & $\begin{array}{l}0.000 \\
(0.357)\end{array}$ & $\begin{array}{l}0.100 \\
(0.142)\end{array}$ & $\begin{array}{l}0.852 \\
(0.000) * * *\end{array}$ & $\begin{array}{l}9.383 \\
(0.670)\end{array}$ & $\begin{array}{l}14.302 \\
(0.282)\end{array}$ & $\begin{array}{l}32.996 \\
(0.000) * * *\end{array}$ & $\begin{array}{l}1.226 \\
(0.268)\end{array}$ & 0.621 \\
\hline
\end{tabular}




\subsection{Rolling Regression Estimation}

The GW-CAPM model is reestimated using the GARCH $(1,1)$ specification and a rolling window of three years. A total of 15 windows are considered. The GRCAPM model is also estimated for Thailand following the model selection results from the last section. The estimates are given in Tables 3 to 8 . The diagnostic results suggest that the models are generally well specified. The ARCH-LM test and Q-statistic for the squared residuals indicate that the problem of ARCH effects has been adequately dealt with. The Jarque-Bera normality test shows no evidence against the normality assumption in most of the cases.

The economic grouping variable is significant in all 15 windows as well as the full sample period for all the five markets. In contrary, the world factor, (and regional factor for Thailand) is not always significant. For Singapore, however, the exposure to world risk is significant for all the windows. For the other four markets, it is interesting to note that the world market returns are not significant in many instances prior to and during the 1997 crisis.

The betas obtained from the rolling estimates of the GW-CAPM model are plotted in Figure 2. These estimates are generally not stable, thus supporting the use of rolling windows. Singapore is the only market with stable betas, and instability is only observed around the 1997-8 financial crisis years. Bigger fluctuations are seen in the other markets, in particular, Indonesia. It is also clear that the financial crisis caused higher instability in beta estimates of four markets.

Figure 2: World and Economic Group Betas of the GW-CAPM Model

Indonesia

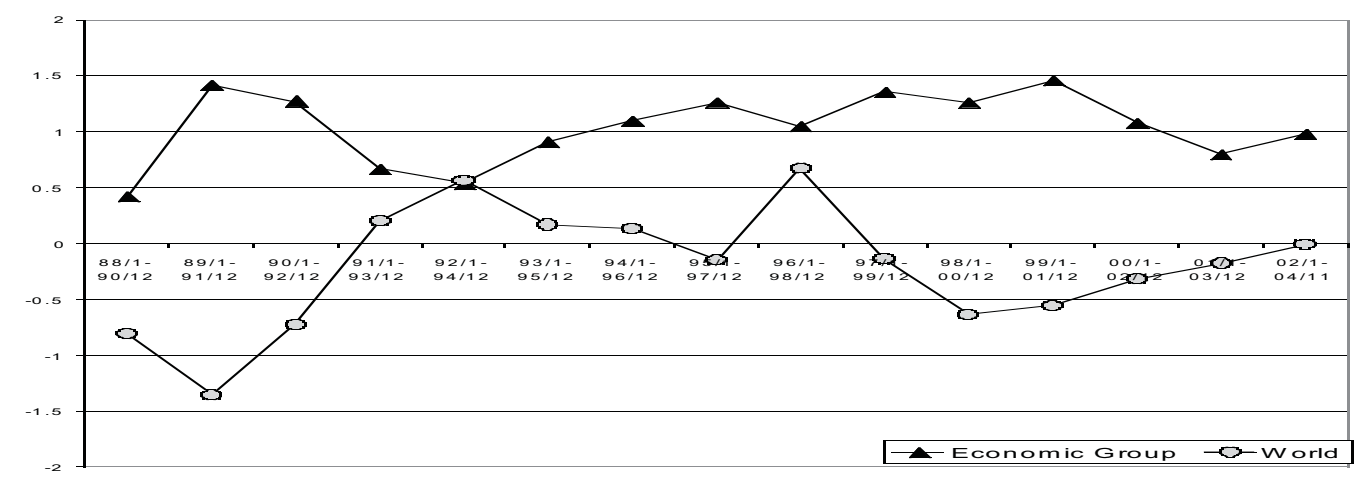

Malaysia

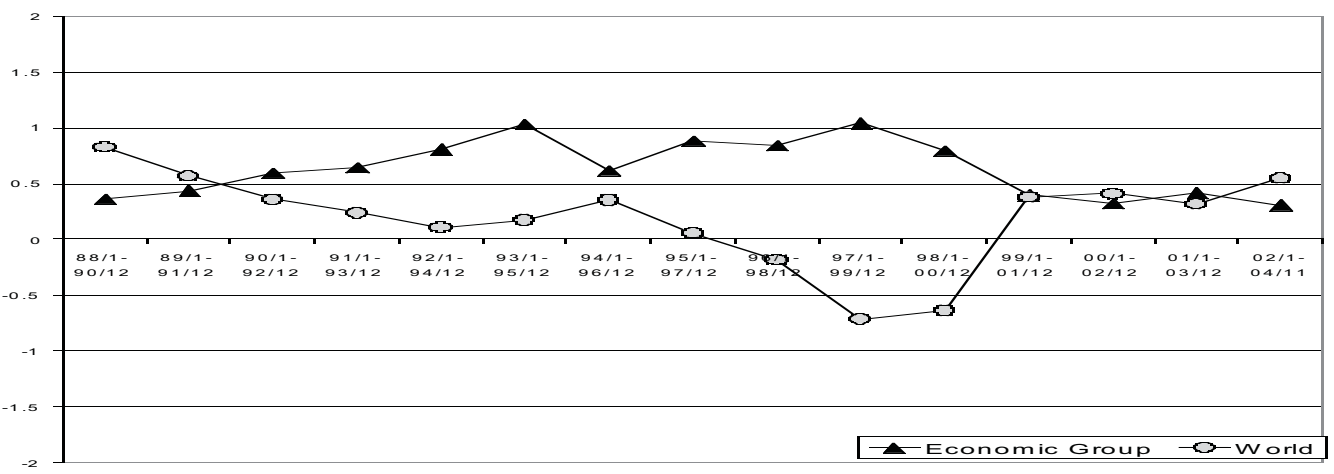


Figure 2: World and Economic Group Betas of the GW-CAPM Model (continued)

Philippines

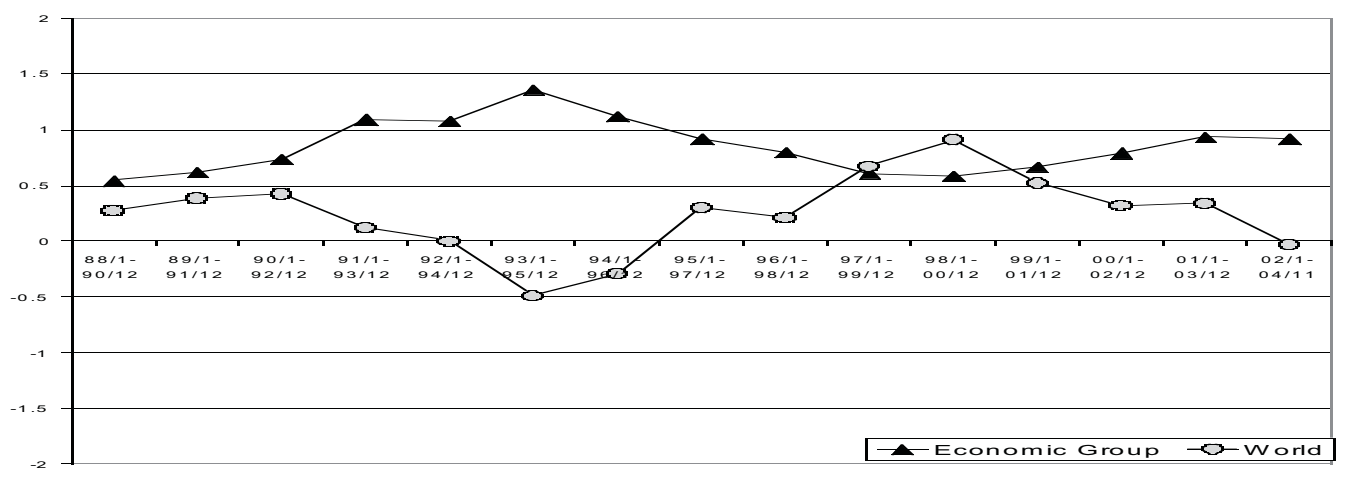

Singapore

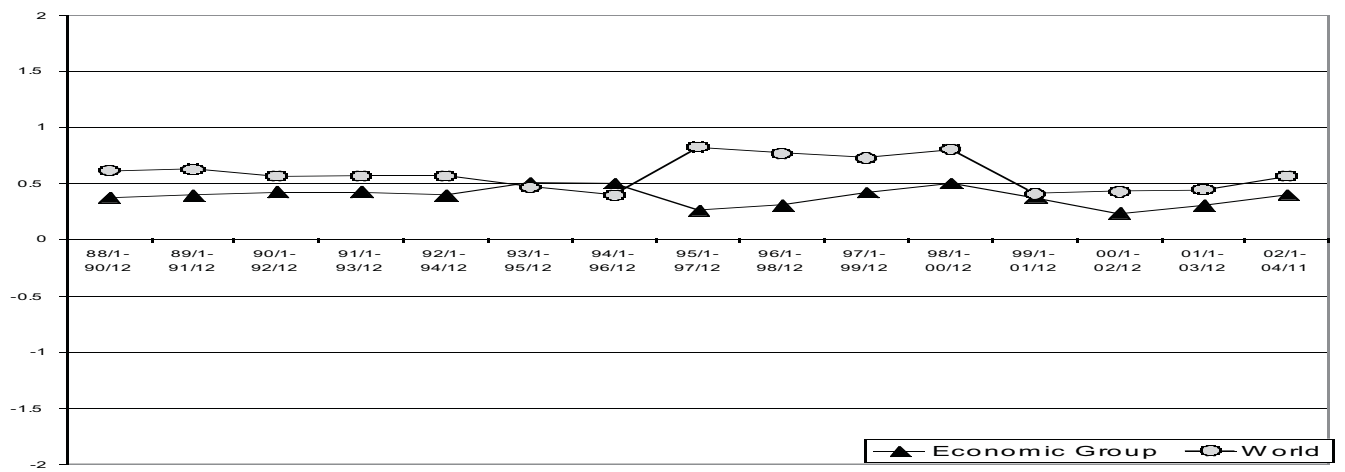

Thailand

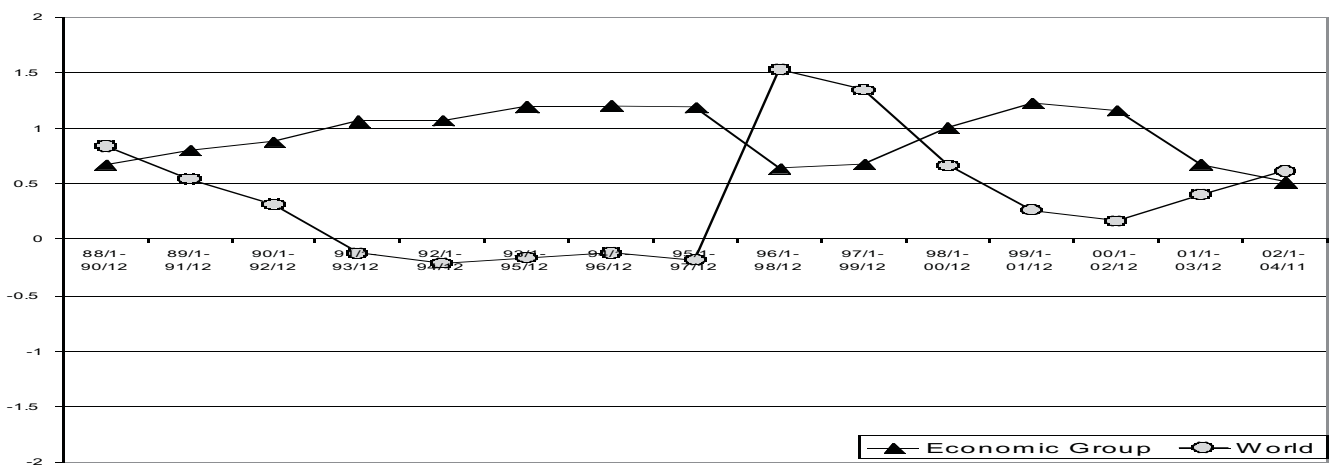

Risk exposures to the economic grouping and world generally move in opposite direction. Markets with low world betas are likely to have a relatively high exposure to economic grouping risks, and vice versa. Specifically, the exposure to the economic grouping factor is relatively higher in Indonesia, Malaysia, Philippines and Thailand except for a small number of windows. The Singapore market has a different behaviour, where the exposure to the world factor is more prominent. Nevertheless, the economic grouping beta for Singapore is only 
slightly lower than the world beta, suggesting that the influence of the former cannot be overlooked.

The five markets have different reactions to the financial crisis. While Malaysia and Indonesia markets saw a drop in exposure to the world risk, the magnitude of the world beta for the other three markets has increased during around the financial crisis period.

These three markets re-anchored themselves to the global market but returned to the pre-crisis position after 1999. The case of Malaysia, however, is different. The exposure to the economic grouping risk has dropped since the crisis. This supports the evidence provided by Goh et al. (2005) that degree of exogeneity of the Malaysia market has increased within the ASEAN group since the implementation of the capital control policy by the Malaysian government in the last quarter of 1998. They show a reduction in the contemporaneous movements between the Malaysia and the other four ASEAN stock markets since the crisis.

\section{Conclusion}

This article tested different specifications of the ICAPM model and proposed the economic grouping factor as an additional variable in the asset pricing. Using data on five stock markets of ASEAN, the importance of exposure to systematic risks in this economic group is shown. We found that the economic grouping factor, if included, increases the explanatory power of the ICAPM model. Some evidence is found that the exposure to world and regional risks has reduced in importance when the economic grouping risks are taken into account.

This article also shows that the pricing mechanism is not stable over time. The Singapore market, which is perhaps most developed market, exhibited relatively stable pricing behaviour compared to any of the other four other markets (Indonesia, Malaysia, Philippines and Thailand). The exposure to world risk is generally higher in the Singapore market, but the other four markets have higher exposure to the economic grouping risk. However, the effect of the economic grouping factor on the Singapore market is rather sizeable and not negligible.

Given that exposure to economic grouping behaviour is not be neglected in international asset pricing model, the findings offer some explanation for segmentation in emerging markets. The higher exposure to movement in returns of the economic group comes together with the reduction in the impact of global market movements on the individual markets, and hence there is lower degree of integration into the world market.

Author statement: The submitting author is Kim-leng Goh: E-mail: klgoh@ um.edu.my. Author statement: The submitting author is Kim-leng Goh: Email: klgoh@um.edu.my at the University of Malaya. The authors express their gratitude for the comments of the reviewer and the assistance of the editors to expedite the copy editing of the paper. The authors are jointly responsible for any remaining errors. 


\section{References}

Akdogan, H. (1992). "Behaviour of systematic risk in a regionally integrated model for stock prices", Economics Letters, Vol 39: 213-216.

Bekaert, K.G., Harvey, C.R. and Ng, A. (2005). "Market integration and contagion", Journal of Business, Vol 78: 39-69.

Bollerslev,T.(1986). “Generalized autoregressiveconditional heteroskedasticity”, Journal of Econometrics, Vol 31: 307-327.

Bollerslev, T. and Wooldridge, J.M., (1992). "Quasi-maximum likelihood estimation and inference in dynamic models with time varying covariances", Econometric Reviews, Vol 11: 143-172.

Brown, R.L., Durbin, J. and Evans, J. M., (1975). "Techniques for testing the constancy of regression relationships over time", Journal of the Royal Statistical Society, Series B, Vol 37: 149-192.

Campbell, J.Y. and Hamao, Y., (1992). "Predictable stock returns in the United States and Japan: a study of long-term capital market integration", Journal of Finance, Vol 47: 43-69.

Chen G.M., Firth, M. and Rui, O.M., (2002). "Stock market linkages: evidence from Latin America", Journal of Banking and Finance, Vol 26: 11131141.

Davidson, S., Faff, R. and Hillier, D., (2003). "Gold factor exposures in international asset pricing”, Journal of International Financial Markets, Institutions \& Money, Vol 13: 271-289.

Engle, R.F. and Ng, V.K., (1993). "Measuring and testing the impact of news on volatility", Journal of Finance, Vol 48: 1749-1778.

Frankel, J.A. and Wei, S.J., (1998). "Open regionalism in a world of continental trade blocs", IMF Working Paper, No. WP/98/10.

Frankel, J.A., Stein, E. and Wei, S.J., (1995). "Trading blocs and the Americas: the natural, the unnatural, and the super-natural", Journal of Development Economics, Vol 47 (No 1): 61-96.

Fratzscher, M., (2002). "Financial market integration in Europe: on the effects of EMU on stock markets", International Journal of Finance and Economics, Vol 7: 165-193.

Goh, K.L., Wong, Y.C. and Kok, K.L., (2005). "Financial crisis and intertemporal linkages across the ASEAN-5 stock markets", Review of Quantitative Finance and Accounting, Vol 24: 359-377.

Johnson, R. and Soenen, L., (1993). "Stock market reaction to EC economic and monetary intention", European Management Journal, Vol 11 (No 1): 85-92.

Johnson, R. and Soenen, L., (2003). "Economic integration and stock market comovement in the Americas", Journal of Multinational Financial Management, Vol 13: 85-100.

Johnson, R., Lindvall, J. and Soenen, L., (1994). "EC economic and monetary integration: implications for European equity investors", European Management Journal, Vol 12 (No 1): 94-101. 
Lessard, D.R., (1973). "International portfolio diversification: A multivariate analysis for a group of Latin American countries", Journal of Finance, Vol 28: 619-633.

Rillo, A.D., (2004). "Trade and financial integration: is there a link?", ASEAN One, November 7-8.

Ripley, D.M., (1973). "Systematic elements in the relationships of national stock market indices", Review of Economics and Statistics, Vol 55: 356-361.

Soydemir, G., (2000). "International transmission mechanism of stock market movements: evidence from emerging equity markets", Journal of Forecasting, Vol 19: 149-176. 